\title{
Perpendicular space accounting of localized states in a quasicrystal
}

\author{
Murod Mirzhalilov (1) and M. Ö. Oktel ${ }^{*}{ }^{*}$ \\ Department of Physics, Bilkent University, Ankara 06800, Turkey
}

(Received 29 April 2020; accepted 16 August 2020; published 26 August 2020)

\begin{abstract}
Quasicrystals can be described as projections of sections of higher dimensional periodic lattices into real space. The image of the lattice points in the projected-out dimensions, called the perpendicular space, carries valuable information about the local structure of the real space lattice. In this paper, we use perpendicular space projections to analyze the elementary excitations of a quasicrystal. In particular, we consider the vertex tightbinding model on the two-dimensional Penrose lattice and investigate the properties of strictly localized states using their perpendicular space images. Our method reproduces the previously reported frequencies for the six types of localized states in this model. We also calculate the overlaps between different localized states and show that the number of type-five and type-six localized states which are independent from the four other types is a factor of golden ratio $\tau=(1+\sqrt{5}) / 2$ higher than previously reported values. Two orientations of the same type-five or type-six which are supported around the same site are shown to be linearly dependent with the addition of other types. We also show through exhaustion of all lattice sites in perpendicular space that any point in the Penrose lattice is either in the support of at least one localized state or is forbidden by local geometry to host a strictly localized state.
\end{abstract}

DOI: 10.1103/PhysRevB.102.064213

\section{INTRODUCTION}

Since their discovery in the 1980s [1], quasicrystals have provided unique problems which challenge the connection between order and periodicity in solids. While there has been immense progress in understanding the structural properties of quasicrystalline systems [2-5], elementary excitations in quasicrystals are much less studied [6]. The absence of periodicity, thus quasimomentum, makes it hard to label the eigenstates of a quasiperiodic system. While some fundamental issues have been overcome in one-dimensional models [7-9], the results in two and three dimensions rely mostly on large-scale computations of spectra [10].

There has been a resurgence of interest in quasiperiodic systems due to experiments which can create quasiperiodic order in engineered systems such as photonic crystals [11], polaritonic cavities [12], and, most recently, ultracold atoms $[13,14]$. While the system sizes in these experiments are much smaller compared to quasicrystalline solids, they are free of impurities and provide an opportunity to measure quantities which are not accessible for solids. Similarly, quasicrystalline order may lead to unexplored phases in models which are well understood for periodic systems [15-17]. Particularly cold atom realizations of quasicrystals may soon explore the effects of local quasicrystal structure in strongly interacting systems.

A quasicrystal can be defined as the projection of a segment of a periodic lattice in a higher dimension [18]. While other methods for defining quasiperiodic order exist [4], the projection method has a geometric appeal which provides insight about the quasiperiodic order. The projection property has

\footnotetext{
*oktel@bilkent.edu.tr
}

been successfully applied to calculate the x-ray diffraction spectra [2,3] and other observables related to structure of quasicrystalline systems [19]. However, the investigation of eigenstates have mostly relied on numerical calculations in periodic approximants to quasicrystals [10] or on the selfsimilar properties obtained through inflation-deflation transformations [7]. The perpendicular space images of eigenstates in the one-dimensional Fibonacci chain [20] and spin models on quasicrystals [21] have been investigated. Perpendicular space images have been calculated for particular eigenstates satisfying an ansatz [22] for two-dimensional tight-binding models [23].

The perpendicular space image of a lattice point carries valuable information about the local connectivity of that point in real space [24]. In general, points which are close to each other in perpendicular space have similar local environments, which maps a particular local lattice configuration to a volume in perpendicular space. As projection from higher dimension is a linear mapping, volumes in perpendicular space are proportional to frequencies of corresponding lattice configurations. In this paper, we apply this general idea to label and count localized eigenstates for a specific quasicrystalline model.

We consider the vertex tight-binding model on a Penrose lattice (PL) [25]. PL can be described as the projection of the intersection of a five-dimensional simple cubic lattice with a 2D plane [18]. The real-space lattice points have orientational order where nearest neighbors are in one of the five starvector directions. Nearest-neighbor bonds define thick and thin rhombuses, which can alternatively be used to define the PL through local matching rules. A vertex tight-binding lattice considers an $s$ state at each point of the PL and uniform hopping probability only over the nearest-neighbor bonds. 
This model was investigated in 1985 where numerical results indicated a density of states peak at zero energy [26,27], which was shown to emerge from strictly localized states (LSs) by Kohmoto and Sutherland [28]. In a following publication, Arai et al. reported six independent types of such strictly LSs and their frequencies [29]. The method for establishing the independence of these states and counting their frequencies relied on identification of real-space structures such as "one three edge rhombus strings" and "bridge sites" combined with inflation-deflation counting of local structures [30].

Here, we show that perpendicular space methods can be used to uniquely label, as well as count, the frequencies of the strictly LSs in this model. This labeling allows us to calculate the overlaps between different LSs and establish the independence of different LSs. While each one of the type-1 to type-four states are independent of each other, as a collective set this is not true for type-5 and type- 6 states. A combination of one type-5 state with a particular type- 2 and a particular type-3 state results in another type-5 state around the same $S 5$ vertex. Thus, the frequencies of type- 5 states which are independent of each other is a factor of golden ratio $\tau=(1+\sqrt{(5)}) / 2$ higher than frequency of type-5 states independent of the collective set of type- 1 to type-5 states. A similar situation plays out for type-6 states. Perpendicular space methods also help identify sites which are forbidden by local connectivity from hosting an eigenstate of zero energy. We show that a finite number of iterations in perpendicular space rules out $f_{\text {forbidden }}=1358-839 \tau=46.95 \%$ of PL vertices from hosting strictly LSs. This process allows us to prove that a site is either forbidden to host a LS or in the support of at least one LS.

The next section contains the basic definitions related to the PL, its perpendicular space, and the vertex model. In Sec. III, we identify the perpendicular space areas corresponding to the six types of LSs and count their frequencies. The overlaps between different LSs are calculated and their independence is established in Sec. IV. The following section details the identification and counting of forbidden sites and shows that each site is either forbidden or in the perpendicular space are of a LS. We close the paper with a summary of our results and conclusions in Sec. VI.

\section{PENROSE LATTICE AND ITS PERPENDICULAR SPACE}

There are various equivalent methods for defining the quasiperiodic PL. Here, we use the projection definition following de Bruijn [18] as our method relies on the perpendicular space of the lattice. Consider the five-dimensional real space as spanned by the orthogonal unit vectors $\hat{u}_{0}, \hat{u}_{1}, \hat{u}_{2}, \hat{u}_{3}, \hat{u}_{4}$ :

$$
\vec{x}=x_{0} \hat{u}_{0}+x_{1} \hat{u}_{1}+x_{2} \hat{u}_{2}+x_{3} \hat{u}_{3}+x_{4} \hat{u}_{4} .
$$

This five-dimensional space can be partitioned into open unit cubes $k_{m}-1<x_{m}<k_{m}$ where $k_{m}$ are integers for $m=$ $0, \ldots, 4$. If we define $\zeta=e^{i \frac{2 \pi}{5}}$, the following five orthogonal vectors also span the five-dimensional space:

$$
\vec{c}_{0}=\sum_{m} \hat{u}_{i}
$$

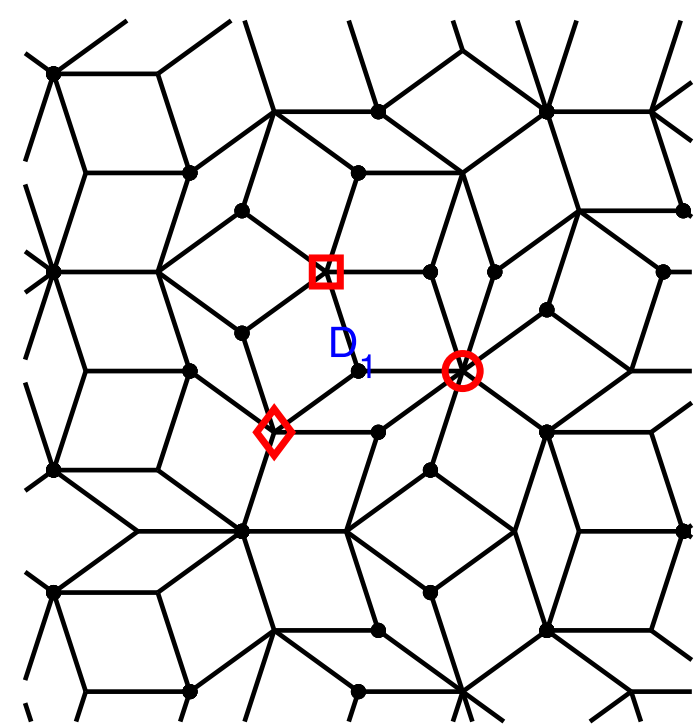

FIG. 1. A finite section of the Penrose lattice in real space. The central point $D_{1}$ has three neighbors. The perpendicular space projections of $D_{1}$ and neighbors are shown in Fig. 2.

$$
\begin{aligned}
& \vec{c}_{1}=\sum_{m} \operatorname{Re}\left(\zeta^{m}\right) \hat{u}_{m}, \\
& \vec{c}_{2}=\sum_{m} \operatorname{Im}\left(\zeta^{m}\right) \hat{u}_{m}, \\
& \vec{c}_{3}=\sum_{m} \operatorname{Re}\left(\zeta^{2 m}\right) \hat{u}_{m}, \\
& \vec{c}_{4}=\sum_{m} \operatorname{Im}\left(\zeta^{2 m}\right) \hat{u}_{m} .
\end{aligned}
$$

We define a two-dimensional plane with the following three equations:

$$
\begin{aligned}
\vec{x} \cdot \vec{c}_{0} & =0, \\
(\vec{x}-\vec{\gamma}) \cdot \vec{c}_{3} & =0, \\
(\vec{x}-\vec{\gamma}) \cdot \vec{c}_{4} & =0 .
\end{aligned}
$$

The intercept vector $\vec{\gamma}=\sum_{m} \gamma_{m} \hat{u}_{m}$ is chosen to be perpendicular to $\vec{c}_{0}$.

A real-space projection of a point in the five-dimensional cubic lattice $\vec{R}_{5}=\sum_{m} k_{m} \hat{u}_{m}$ is in the PL if and only if there is a point in its open unit cube which satisfies Eqs. (3). We further assume that $\vec{\gamma}$ is chosen such that the plane and the cube intersection does not yield a singular lattice [18]. The points $k_{0}, \ldots, k_{4}$ satisfying this condition can be expressed as

$$
\vec{R}_{5}=\sum_{m} k_{m} \hat{u}_{m}=\frac{2}{5}\left(x_{R} \vec{c}_{1}+y_{R} \vec{c}_{2}+x_{\perp} \vec{c}_{3}+y_{\perp} \vec{c}_{4}+\frac{N_{0}}{2} \vec{c}_{0}\right) .
$$

We refer to $\vec{r}_{R}=x_{R} \hat{i}+y_{R} \hat{j}$ as the real-space projection and $\vec{R}_{\perp}=x_{\perp} \hat{i}_{\perp}+y_{\perp} \hat{j}_{\perp}$ as the perpendicular space image of this point. The unit orthogonal vectors spanning the real space are defined as $\hat{i}, \hat{j}$ and similarly perpendicular space is constructed by $\hat{i}_{\perp}, \hat{j}_{\perp} \cdot N_{0}=\vec{R}_{5} \cdot \vec{c}_{0}=\sum_{m} k_{m}$ is an integer by construction and is called the index of this point.

A finite section of the PL is shown in Fig. 1, where we can equivalently express the positions $\vec{r}_{R}=\sum_{m} k_{m} \hat{e}_{m}$ where 


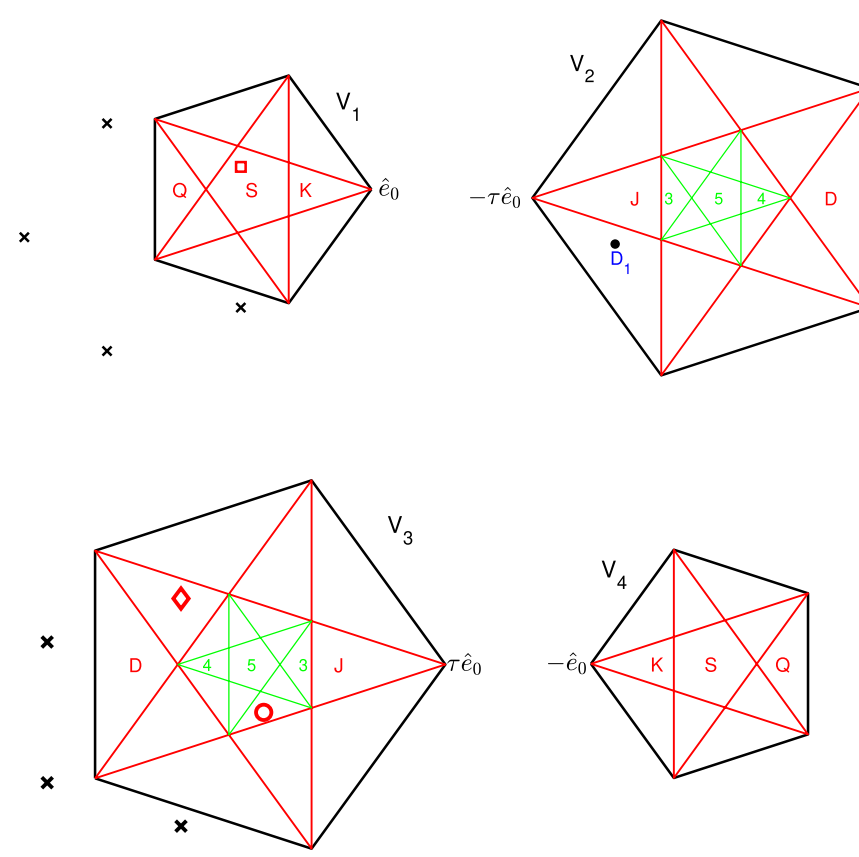

FIG. 2. The four pentagons $V_{1}$ to $V_{4}$ in perpendicular space. Notice that $V_{1}$ and $V_{4}$ are scaled up for visual clarity on all subsequent figures. Perpendicular space image of $D_{1}$ and and its neighbors are shown. The points marked outside the pentagons $V_{1}$ and $V_{3}$ differ from $D_{1}$ by star vectors $\hat{e}_{m}$ but have no counterparts in real space as they lie outside the pentagons. The regions for $S-, Q-, K-, S 5-$, $S 4$-, S3-, $D$-, J-type vertices are marked by S, Q, K, 5, 4, 3, D, J, respectively; empty regions have the same vertex type as the marked region they are related by fivefold rotational symmetry.

the five star vectors are $\hat{e}_{m}=\operatorname{Re}\left(\zeta^{m}\right) \hat{i}+\operatorname{Im}\left(\zeta^{m}\right) \hat{j}$. Thus all the nearest neighbors are aligned in the directions $\pm \hat{e}_{m}$ and there is orientational order throughout the PL.

The index $N_{0}$ can only take four values $N_{0}=1,2,3,4$, and the projection conditions Eqs. (3) define a finite area $V_{N_{0}}$ which constrains the perpendicular space image of points in the PL. These areas form four regular pentagons in perpendicular space and are shown in Fig. 2. For indices $N_{0}=1$, 4, the pentagons have circumscribed circles of radius 1 , while $N_{0}=$ 2,3 have circumscribed circles of radius $\tau=(1+\sqrt{(5)}) / 2$. We refer to the radius of the circumscribed circle of a pentagon as the radius of the pentagon throughout the paper. The collection of these four pentagons $V_{1}, V_{2}, V_{3}, V_{4}$ form all of the perpendicular space of the PL. Although the perpendicular space is three-dimensional, the image of all PL points are restricted to these four two-dimensional regions $[31,32]$. This fact shows that the projection could be carried out from an initial four-dimensional lattice [33], but we choose not to use such an approach.

It is important to note that the perpendicular space image of any point contains information about the local structure of the point in real space [24]. Consider point $D_{1}$ in Fig. 1 and its perpendicular space image $D_{1}$ in Fig. 2 . We see that this point has index 2 as it is in the $V_{2}$ pentagon. Any nearest neighbor of $D_{1}$ can be along either the $+\hat{e}_{m}$ or the $-\hat{e}_{m}$ directions. If a point is reached by a positive $\hat{e}_{m}$ vector from $D_{1}$, its perpendicular space image must lie in $V_{3}$ and if by a negative $\hat{e}_{m}$ in $V_{1}$. Furthermore, the perpendicular space position of the neighbor is related to which $\hat{e}_{m}$ vector is used in real-space translation. From Eqs. (2), we find the correspondence

$$
\hat{e}_{0} \rightarrow \hat{e}_{0}, \quad \hat{e}_{1} \rightarrow \hat{e}_{2}, \quad \hat{e}_{2} \rightarrow \hat{e}_{4}, \quad \hat{e}_{3} \rightarrow \hat{e}_{1}, \quad \hat{e}_{4} \rightarrow \hat{e}_{3}
$$

between real-space translations and perpendicular space translations. All five possible perpendicular space points reached from $D_{1}$ with positive $\hat{e}_{m}$ are shown in Fig. 2. Only two of these are inside $V_{3}$, thus $D_{1}$ has only two neighbors of index 3. A similar reasoning for negative $\hat{e}_{m}$ translations show that only one out of a possible five neighbors is in $V_{1}$, thus $D_{1}$ has only three neighbors in total.

The nearest-neighbor configurations can be used to classify vertices and we use the nomenclature of de Bruijn [18]. $K$, $Q$, and $S$ vertices have index of $N_{0}=1,4$ and their perpendicular space regions in $V_{1}, V_{4}$ are shown in Fig. 2. Similarly $D, J, S 3, S 4$, and $S 5$ vertex regions are marked in $V_{2}, V_{3}$. All the points of the PL have a perpendicular space image in one of the four $V_{m}$ pentagons. The mapping from PL points to perpendicular space is not one to one but injective; there are some points inside the $V_{m}$ pentagons which do not correspond to any PL point. As an example, consider the points on the lines separating regions for distinct vertex types. A point on this line does not correspond to a point of either vertex type, it remains unassociated with any PL point as $\tau$ is an irrational number. Still, the points in perpendicular space associated with PLs are dense; any such point has infinitely many similar points in any finite neighborhood. The set of points inside the pentagons is a two-dimensional analog of the real line with all rational numbers removed.

The mapping from five-dimensional space to real and perpendicular spaces Eqs. (2) is a linear transformation. Thus, not only are the PL-projected points inside the pentagons dense, their density is uniform inside all four pentagons. The ratio of areas in perpendicular space give the ratio of corresponding vertex sites in real space. As an example, consider the frequency of $S$ vertices in the PL. We see that the area corresponding to $S$ in $V_{1}$ is a pentagon of radius $\tau^{-2}$. As $V_{1}$ and $V_{2}$ have radii of 1 and $\tau$ the ratio of the area of $S$ regions to total area of pentagons is

$$
f_{S}=\frac{\tau^{-4}}{1+\tau^{2}}=\frac{18-11 \tau}{5} \simeq 0.04032
$$

as found in the literature [32]. We use the same reasoning to count and classify the LS in the following.

The vertex tight-binding model is defined on the PL with uniform hopping strength on all nearest-neighbor bonds,

$$
\mathcal{H}=-\sum_{<i j>}\left|\vec{R}_{i}\right\rangle\left\langle\vec{R}_{j}\right|
$$

where the sum is only on neighbors which are connected with $\hat{e}$ vectors and $|\vec{R}\rangle$ is a state localized at the PL real-space point $\vec{R}$ which form an orthonormal set $\left\langle\vec{R} \mid \vec{R}^{\prime}\right\rangle=\delta_{\vec{R} \cdot \vec{R}^{\prime}}$. The hopping strength sets the energy scale and by taking it as 1 we report dimensionless quantities throughout the paper. The eigenstates $|\Psi\rangle=\sum \Psi(\vec{R})|\vec{R}\rangle$ satisfy

$$
\mathcal{H}|\Psi\rangle=E|\Psi\rangle .
$$


The spectrum of this problem has only been calculated numerically. Initial numerical work on finite systems showed that the density of states near zero energy had a significant peak $[26,27]$. The reason for the zero-energy peak was clearly identified by Kohmoto and Sutherland-the vertex model has an infinitely degenerate manifold of strictly LSs [28]. These eigenstates have a nonzero wave function only on a finite number of sites of the PL and are confined by local connectivity. Destructive interference between different sites with nonzero wave function prevents probability leakage to any neighboring sites. We consider only these states with zero energy throughout the rest of the paper, and refer to them as LSs for convenience; there should not be confusion with eigenstates which are exponentially localized.

The PL is bipartite, the vertices with index 1,3 have only nearest neighbors with indices 2 or 4 . Thus, the vertex model Eq. (7) has a spectrum which is symmetric under a sign change of energy. The LSs have zero energy and bipartite symmetry enables us to split the degenerate manifold of LSs into states which are defined on the (1-3) sublattice and states defined on the (2-4) sublattice. As there are an equal number of these states, we consider the (1-3) sublattice in all our calculations. All LSs we display on $V_{1}, V_{3}$ have a corresponding state in $V_{4}, V_{2}$ which can be obtained by inversion (both in real and perpendicular space).

Arai et al. reported six independent types of LSs and their frequencies [29]. In the following section, we use perpendicular space projections to label and count these states.

\section{FREQUENCIES AND LABELING OF STRICTLY LOCALIZED STATES}

A strictly LS is an eigenstate of the tight-binding Hamiltonian Eq. (7) which has nonzero density only on a finite number of lattice sites. When the support, i.e., the set of sites with nonzero wave function, is finite, there is a radius $\mathrm{R}$ beyond which the wave function is exactly zero. The condition for obtaining such states is more stringent than the usual definition of LSs which have exponentially decaying tails going off to infinity.

The support of the LS must satisfy certain conditions, any one of the nearest neighbors of a point in the support must be a nearest neighbor of at least one other point in the support. Furthermore, the wave function defined on the support must be such that it must interfere destructively on all the nearestneighbor points. Thus, local configuration and connectivity of sites play a decisive role on the existence of LSs. Strict localization does not take place in periodic lattices unless the LS is confined to a single unit cell. However, similar strict localization can be obtained by imposing an external magnetic field on the lattice and forming flux cages [34].

The vertex model on the PL is bipartite, the sites with index $N_{0}=1,3$ [the (1-3) sublattice] are connected only to sites with index $N_{0}=2,4$ [the (2-4) sublattice]. Thus, the existence of an eigenstate with energy $E>0$ implies that there is another eigenstate with energy $-E$. These two eigenstates can be obtained from each other by multiplying the wave function on one of the sublattices by -1 . All LSs in the vertex model have zero energy, forming a massively degenerate manifold. As the first step, we divide this manifold into two using

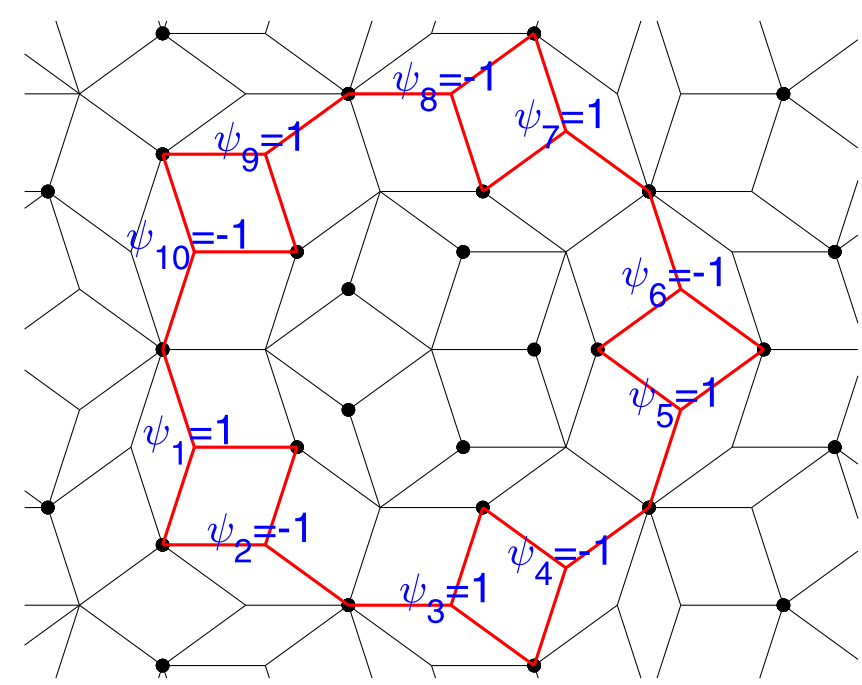

FIG. 3. Type-1 LS support contains ten points forming a ring around an $\mathrm{S}$ vertex. All the points in the support have index 3, points in the (2-4) sublattice are marked with black dots. The wave functions $\psi$ alternate as \pm 1 , all other points in the (1-3) sublattice have zero wave function.

the bipartite symmetry of the PL. If a LS $\Psi_{1}$ has nonzero wave function in both sublattices, it must be degenerate with another LS $\Psi_{2}$ which is obtained by multiplying $\Psi_{1}$ with $(-1)^{N_{0}}$ at each lattice site. The difference $\Psi_{1}-\Psi_{2}$ is a LS defined in the (1-3) sublattice, while the sum $\Psi_{1}+\Psi_{2}$ resides on the (2-4) sublattice. Thus, all LSs can be chosen to lie only in one sublattice.

We present results for states defined on the (1-3) sublattice, thus all the sites in the support of LSs have perpendicular space images in $V_{1}$ and $V_{3}$, while the nearest neighbors are on $V_{2}$ and $V_{4}$. We follow the nomenclature of Ref. [29], who have found six real-space configurations for LSs. We use perpendicular space images of the points in the support of each one of these LSs to count and label them.

A type-1 LS is formed by ten points which form a ring around an $S$ vertex as shown in Fig. 3. Each one of the points in the support are the second-nearest neighbors of two more points in the support and the wave function alternates signs going from one point to its second-nearest neighbor. Now consider the perpendicular space images of all the points in the support as shown in Fig. 4.

In real space, a point in the support is connected to its second-nearest neighbor by a vector $\hat{e}_{i}-\hat{e}_{j}$, the minus sign showing that all the points in the support have the same index. Thus, the vectors connecting the perpendicular space images also have the same form $\hat{e}_{i}-\hat{e}_{j}$, obtained by Eqs. (5). As shown in Fig. 4, the connecting vectors define a decagon with five short and five long sides in perpendicular space. One can imagine moving one of the vertices of this shape will also move all the other vertices if all the connecting vectors are held rigid. As long as all the vertices of the decagon are still inside $V_{3}$, a type-1 LS can be defined on these points. The mapping between real space and perpendicular space is dense and uniform as discussed in the previous section, thus the area covered by moving one of the vertices while keeping all the 


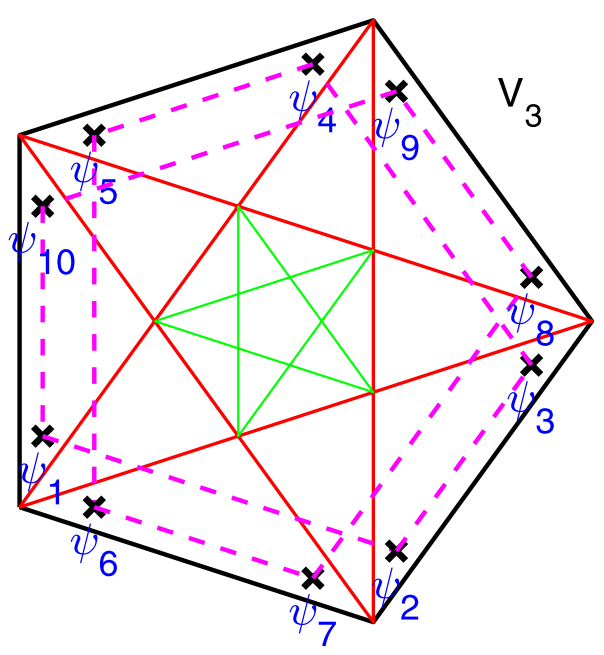

FIG. 4. The perpendicular space images of the points shown in Fig. 3. Nearest neighbors are connected by vectors $\hat{e}_{i}-\hat{e}_{j}$. If all ten points lie in $V_{3}$ as shown in the figure, type-1 LS exists on this set.

vertices inside $V_{3}$ is proportional to the frequency of type-1 states.

The allowed region for the movement of any one of the vertices is shown by the ten identical green pentagons in Fig. 5. These pentagons have radius $\tau^{-4}$, thus the frequency of type- 1 states can be calculated by dividing the area of one of these pentagons with the sum of the areas of $V_{1}$ and $V_{3}$,

$$
f_{T 1}=\frac{\tau^{-8}}{1+\tau^{2}}=\frac{123-76 \tau}{5} \simeq 5.883 \times 10^{-3},
$$

which is the result obtained by Arai et al. [29].

The perpendicular space position of any one of the vertices inside the pentagons can be used to label and distinguish different type-1 states. Equivalently, we can use the perpendicular space position of the central $S$ vertex which lies in $V_{1}$ to label the states. Thus a type- 1 state is uniquely labeled by a vector $\left|T 1, \vec{R}_{\perp}\right\rangle$. Here the vector $\vec{R}_{\perp}$ must lie inside a

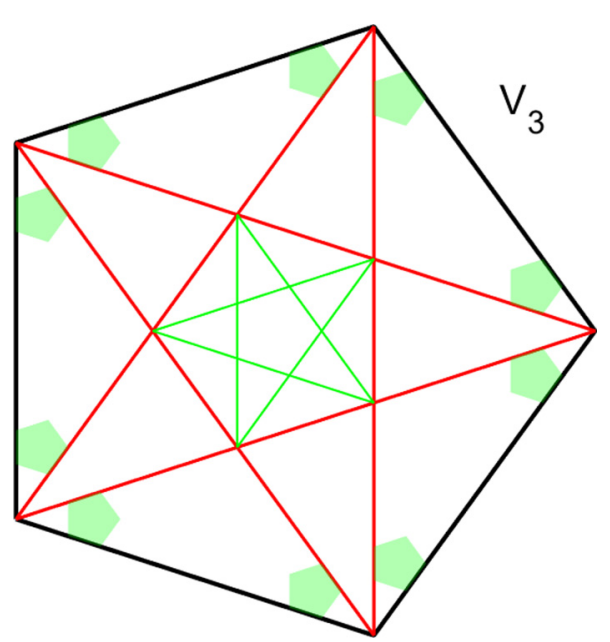

FIG. 5. The allowed area for type-1 vertices. Choosing the position of one vertex in one of the pentagons fixes the position of the remaining vertices as in Fig. 4. Each pentagon has radius $\tau^{-4}$. All vertices in the support are $D$ vertices.

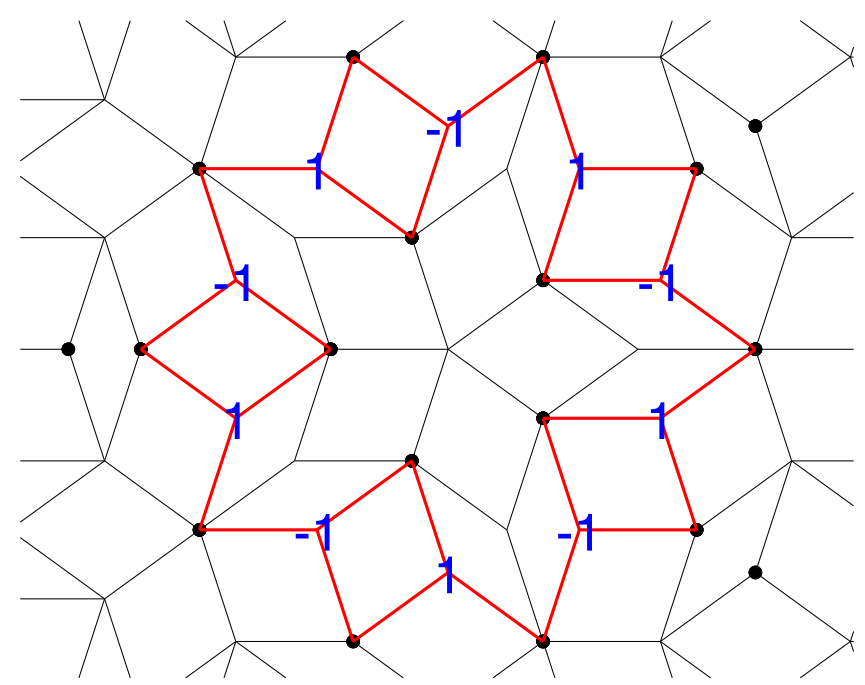

FIG. 6. Type-2 LS has a support of ten points around an $S 5$ vertex. All points in the support have index 3 and are $D$ vertices. The nonzero wave functions are written over the lattice sites.

regular pentagon of radius $\tau^{-4}$ centered at the origin. This region has smaller area than the area of all $S$ vertices, so only $\tau^{-4} \simeq 14.59 \%$ of $S$ vertices have a type-1 state around them. The perpendicular space positions of points in the support can be reached by adding $\hat{e}_{0}+\hat{e}_{2}+\hat{e}_{3}-\hat{e}_{1}$ or $\hat{e}_{0}+\hat{e}_{2}+\hat{e}_{3}-\hat{e}_{4}$ and their fivefold rotations to $\vec{R}_{\perp}$. We should also stress that $\vec{R}_{\perp}$ is not defined on the set of real numbers $\mathbb{R}^{2}$, but any vectors that have rational inner products with star vectors are removed from this set. Nonetheless, this vector uniquely defines the local neighborhood in real space and the density of similar local configurations can be counted as above.

Type-2 LS are similar to type-1 as their support contains ten points arranged in a second-nearest neighbor ring with sign-alternating wave function, see Fig. 6 . However, the ring is centered at an $S 5$ vertex instead of an $S$ vertex. Plotting the perpendicular space positions of the sites in the support, we observe that these points create a regular decagon. Moving this decagon with the constraint that all the vertices stay inside $V_{3}$ defines the allowed areas of type 2. As shown in Fig. 7, the allowed areas are pentagons of radius $\tau^{-3}$. The ratio of the area of one of these pentagons to the total area of $V_{1}$ and $V_{3}$ gives the type- 2 frequency,

$$
f_{T 2}=\frac{\tau^{-6}}{1+\tau^{2}}=\frac{47-29 \tau}{5} \simeq 1.540 \times 10^{-2},
$$

in agreement with Ref. [29]. We label the states once again with a perpendicular space vector $\left|T 2, \vec{R}_{\perp}\right\rangle$, and use the perpendicular space position of the central $S 5$ vertex as $\vec{R}_{\perp}$. Thus, $\vec{R}_{\perp}$ must lie in a regular pentagon of radius $\tau^{-3}$, which exactly is the perpendicular space area of $S 5$ vertices, showing that all $S 5$ vertices have a type-2 LS encircling them. The perpendicular space positions of the sites in the support of the LS can be reached from $\vec{R}_{\perp}$ by adding $-\hat{e}_{0}+\hat{e}_{4},-\hat{e}_{0}+\hat{e}_{1}$ and their fivefold rotations.

Type- 3 LSs are different from type- 1 and type- 2 in two important regards. First, they break the fivefold rotational symmetry, thus five different orientations of type-3 states exist. Second, the support of type-3 contains $2 Q$ vertices 


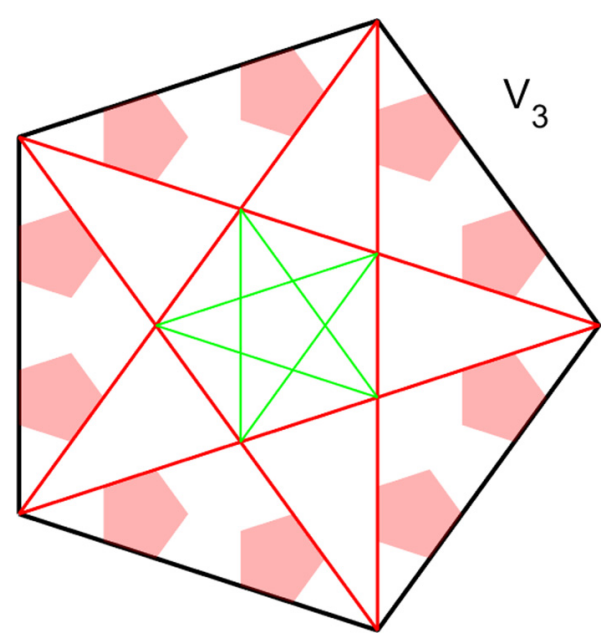

FIG. 7. Allowed areas for the ten points in the support of type-2 LS. Each pentagon is inside a $D$ region and has radius $\tau^{-3}$.

which have index 1 , so the perpendicular space image of the type-3 support has a component in $V_{1}$ as well as $V_{3}$. One orientation of type-3 is displayed in Fig. 8. Once again, one can calculate the perpendicular space image of the 12 points in the support with respect to each other. Moving these points with the constraint that all of them remain in $V_{1}$ and $V_{3}$ define the allowed areas for this particular orientation of type- 3 as shown in Fig. 9. Each one of the 12 regions is a kite with three

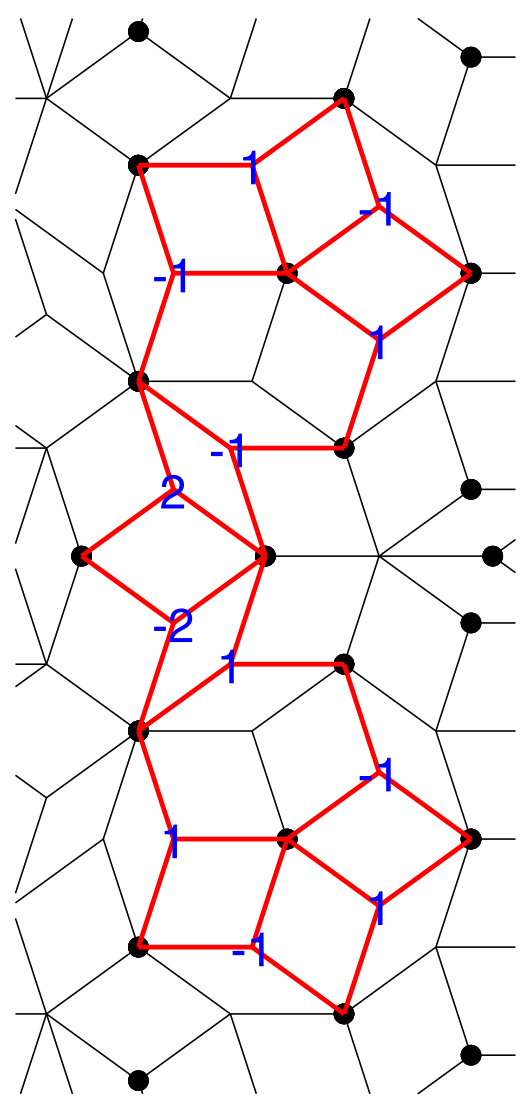

FIG. 8. Type-3 LS with $\hat{e}_{0}$ orientation. There are 12 points in the support, two of which have index 1.
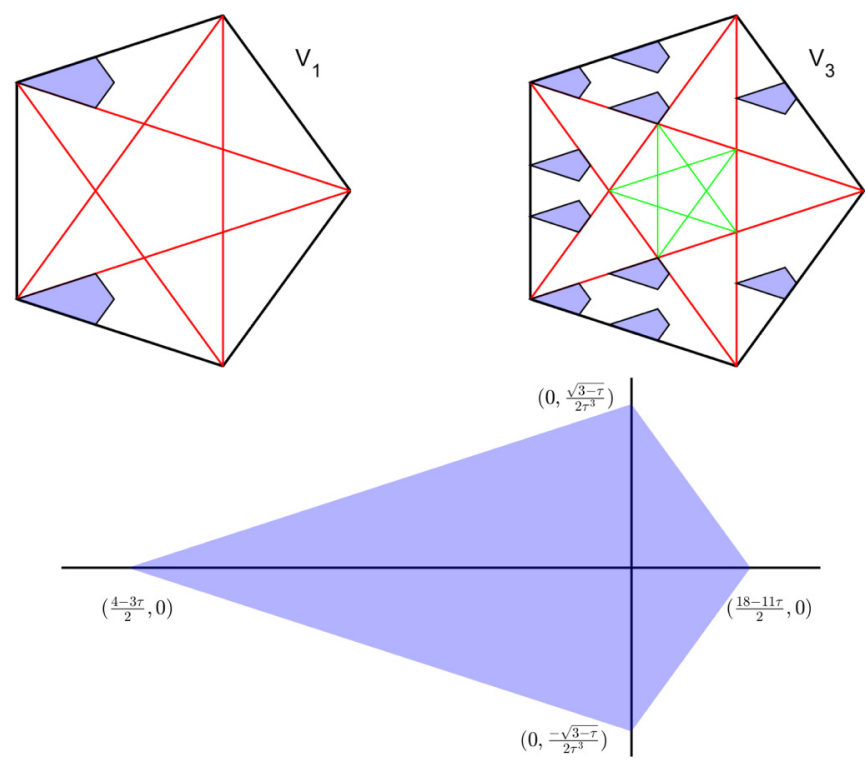

FIG. 9. Allowed areas for the vertices of type- 3 with $\hat{e}_{0}$ orientation. $V_{1}$ is scaled by $\tau$ for visual clarity, all ten kites have the same size. The perpendicular space vector $R_{\perp}$ used to label type-3 vertices is chosen to lie in the kite shown in panel (c).

$\frac{3 \pi}{5}$ and one $\frac{\pi}{5}$ angle. The long diagonal of the kite has length $7-4 \tau$ and the short diagonal $\sqrt{3-\tau} \tau^{-3}$, which gives the fraction of the one particular orientation of type- 3 as

$$
\begin{aligned}
f_{T 3,0} & =\frac{(7-4 \tau) 2 \tau^{-3}}{5 \tau\left(1+\tau^{2}\right)} \\
& =\frac{68-42 \tau}{5} \\
& \simeq 8.514 \times 10^{-3} .
\end{aligned}
$$

All other orientations of type 3 can be obtained from $\hat{e}_{0}$ orientation by $2 \pi / 5$ rotations, and their allowed regions follow the same pattern, only with the correspondence in Eqs. (5). In Fig. 10, we show allowed areas for all the orientations. It can be seen that regions in $V_{3}$ for two different orientations can overlap. The overlap in perpendicular space shows that two different orientations of type 3 in real space can have the same point in their support. The real space overlaps of type 3 can be used to define LSs which have linked second-neighbor support
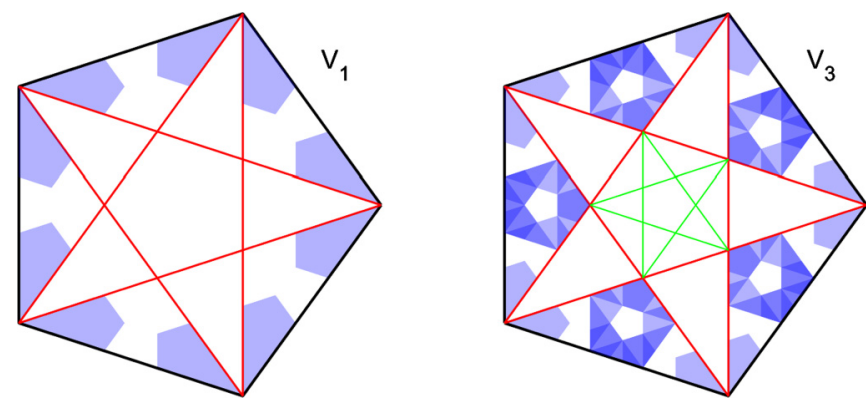

FIG. 10. Allowed areas for all orientations of type-3 states. While eight out of the 12 areas have overlaps between type- 3 states of different orientation, four sites remain unique to each orientation. 


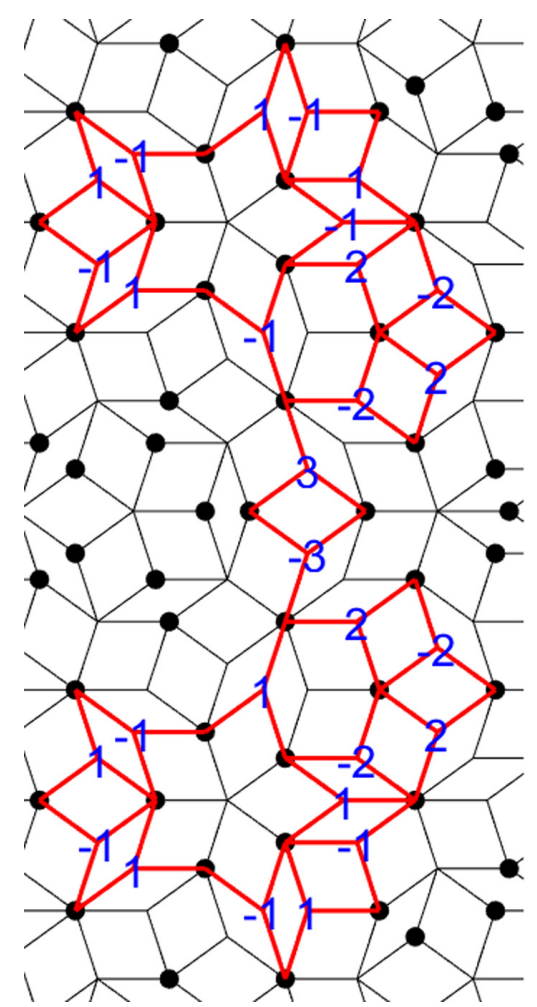

FIG. 11. Type-4 localized state with $\hat{e}_{0}$ orientation.There are 18 sites with index 3 and ten sites with index 1 in the support.

forming longer LSs, as proposed by Kohmoto and Sutherland [28], but such ring states will not be independent from the LS defined here. The independence and overlaps of different LSs will be explored in the next section. Even if different type-3 states are not automatically orthogonal, they remain independent and the total type- 3 fraction is

$$
f_{T 3}=\sum_{m=0}^{4} f_{T 3, m}=68-42 \tau \simeq 4.257 \times 10^{-2},
$$

in agreement with Ref. [29]. Type-3 states can be labeled by their orientation and the relative position of the point within the allowed perpendicular space region $\left|T 3, \hat{e}_{m}, \vec{R}_{\perp}\right\rangle$ where $\hat{e}_{m}$ denotes the orientation and $\vec{R}_{\perp}$ is limited inside the quadrilateral defined by $(4-3 \tau) / 2 \hat{e}_{m},(18-11 \tau) / 2 \hat{e}_{m}$ and $\frac{ \pm 1}{2 \tau^{3}} \sqrt{3-\tau} \hat{z} \times \hat{e}_{m}$, see Fig. 9 .

Type-4 has 28 sites in its support as seen in Fig. 11, and it breaks the fivefold rotational symmetry. The allowed areas for one orientation are displayed in Fig. 12 and one can notice that the allowed area is a scaled version of the type- 3 kite by a factor of $\tau^{-1}$, which allows us to write

$$
f_{T 4,0}=\tau^{-2} f_{T 3,0}=\frac{178-110 \tau}{5} \simeq 3.252 \times 10^{-3} .
$$

Considering all five orientations, we see that there is significant overlap between different orientations of type 4, particularly in $V_{1}$ as seen in Fig. 13. We establish the independence of type- 4 states from previous types and each other in the next
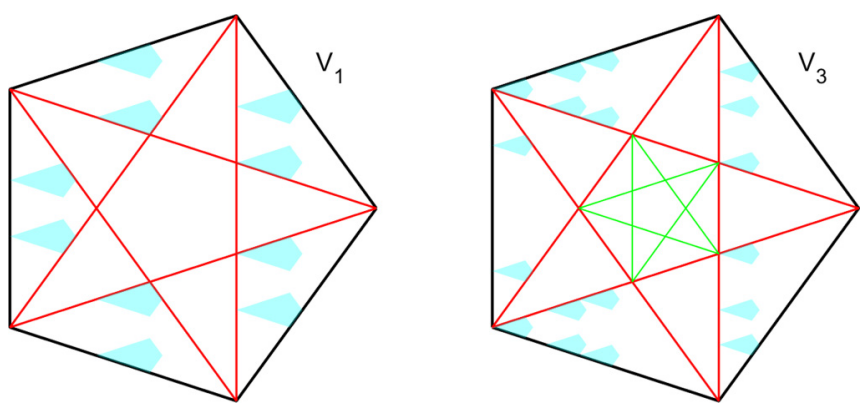

FIG. 12. Allowed areas for the vertices of type-4 LS with $\hat{e}_{0}$ orientation. Each kite is smaller by a factor of $\tau$ compared to the type-3 areas in Fig. 9. The labeling inside the kite is done similarly to type-3 states as in Fig. 9.

section. The total frequency is

$$
f_{T 4}=\sum_{m=0}^{4} f_{T 4, m}=178-110 \tau \simeq 1.626 \times 10^{-2},
$$

in agreement with Ref. [29]. We label type-4 states in a similar manner to type-3, $\left|T 4, \hat{e}_{m}, \vec{R}_{\perp}\right\rangle$, however, the kite constraining the perpendicular space region is smaller by a factor of $\tau$, given by the points $-(7-4 \tau) / 2 \hat{e}_{m},(18 \tau-29) / 2 \hat{e}_{m}$, and $\frac{ \pm 1}{2 \tau^{4}} \sqrt{3-\tau} \hat{z} \times \hat{e}_{m}$.

Type-5 LSs have 19 sites in their support and they break the fivefold symmetry as shown in Fig. 14. These are the only LSs which have a density on an $S 5$ vertex and the triangular area in the $S 5$ region shown in Fig. 15 is used to label them. Plotting all five possible orientations in Fig. 16, we see that not all $S 5$ vertices support a type-5 LS. An $S 5$ site can support one type-5 LS or two different orientations of type 5. The allowed region for the state $\left|T 5, \hat{e}_{0}, \vec{R}_{\perp}\right\rangle$ with $\hat{e}_{0}$ orientation is a triangle with vertices at $\hat{e}_{0} / \tau^{3}, \hat{e}_{1} / \tau^{3}$ and $\hat{e}_{4} / \tau^{3}$, which gives an area

$$
f_{T 5,0}=\frac{1}{5 \tau^{8}}=\frac{34-21 \tau}{5} \simeq 4.257 \times 10^{-3} .
$$

When we consider all orientations of the type- 5 states, the frequency becomes

$$
f_{T 5}=5 f_{T 5,0}=\frac{1}{\tau^{8}}=34-21 \tau \simeq 2.128 \times 10^{-2} .
$$
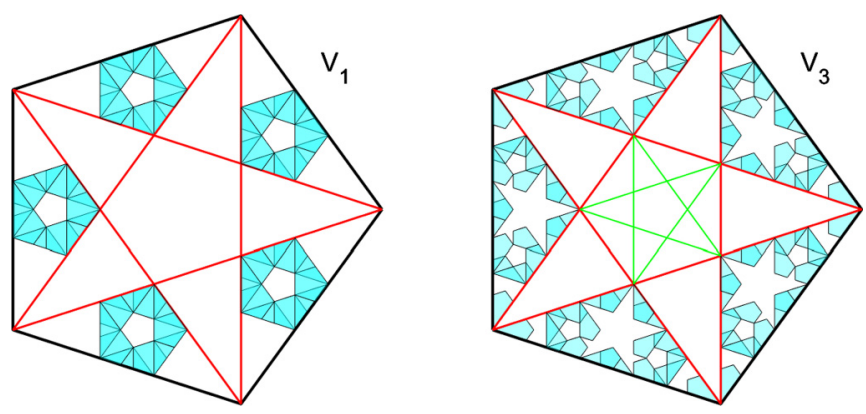

FIG. 13. Allowed areas for Type-4 LS of all orientations superimposed. There are only two unique sites for each orientation which are in $V_{3}$. 


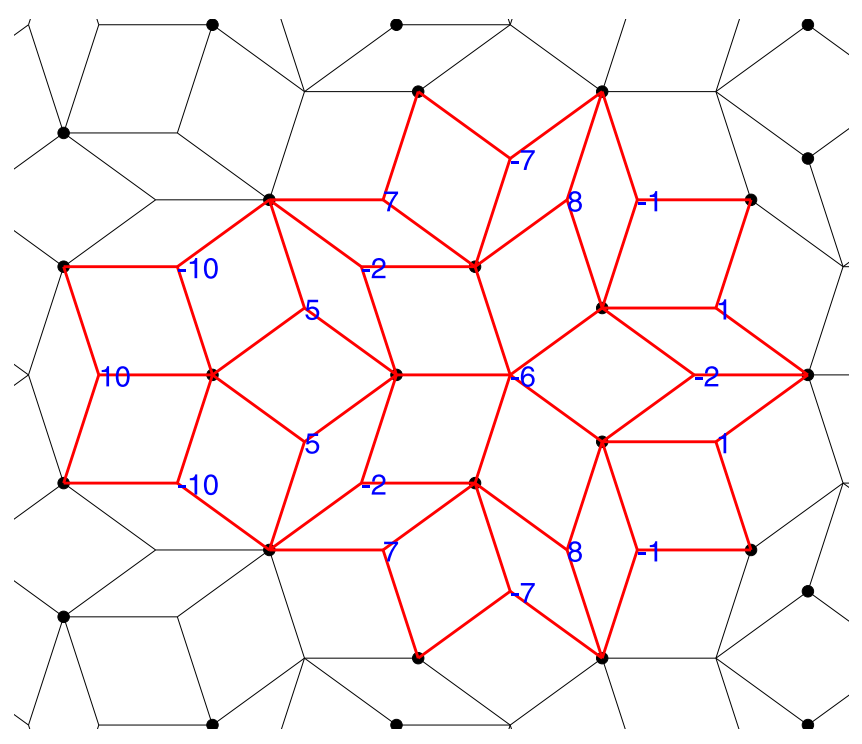

FIG. 14. Type-5 LS with $\hat{e}_{0}$ orientation in real space. Five of the sites in the support have index 1 , while the remaining 14 have index 3 .

This expression is a factor of $\tau$ larger than the value reported in Ref. [29]. This frequency gives us the number of type-5 states that are independent of each other, but in the next section we show that when other types of LSs are taken into account, two type- 5 states sharing the same $S 5$ vertex are not independent from each other. The number of type- 5 states that are independent from each other and types 1-4 is in agreement with the previously reported value.

Finally, the real-space configuration for type-6 is displayed in Fig. 17 and corresponding perpendicular space pictures of one orientation and all five orientations are given in Figs. 18 and 19, respectively. Type-6 LS is the only LS with support containing an $S$ site. Only some of the $S$ sites which have type1 LSs around them can support type-6 LSs. Similar to type 5, some $S$ sites support only one orientation of type 6 while some of them support two different orientations. The allowed areas are triangles which can be obtained by scaling type- 5 allowed area with $\tau$, so type-6 LSs $\left|T 6, \hat{e}_{0}, \vec{R}_{\perp}\right\rangle$ with $\hat{e}_{0}$ orientation must have $\vec{R}_{\perp}$ lie in a triangle with vertices at $\hat{e}_{0} / \tau^{4}, \hat{e}_{1} / \tau^{4}$,
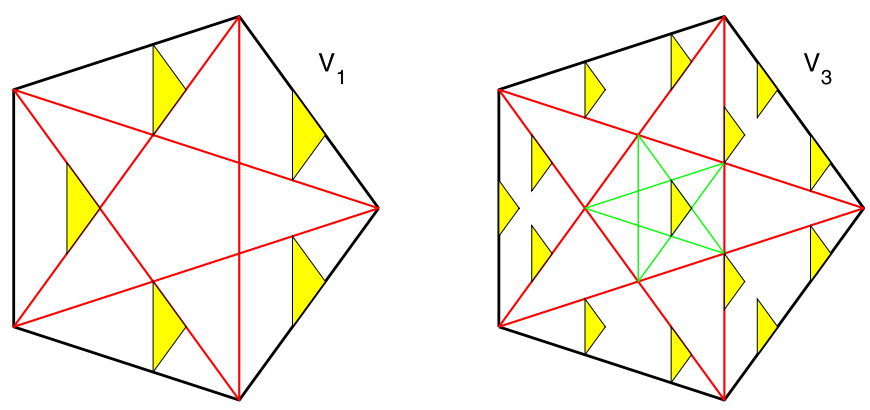

FIG. 15. Allowed areas for vertices of type-5 LS with $\hat{e}_{0}$ orientation are triangles. The central area in $V_{3}$ is used to label type-5 states.
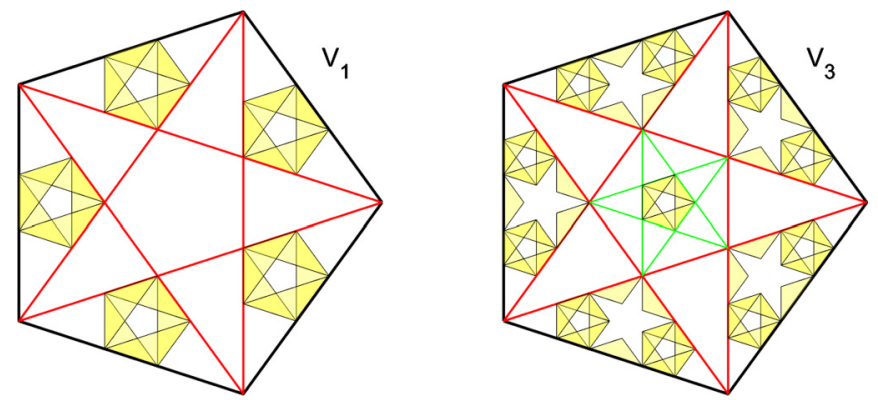

FIG. 16. Allowed areas for all orientations of type 5. Notice that two allowed areas for different orientations can overlap in both $V_{1}$ and $V_{3}$.

and $\hat{e}_{4} / \tau^{4}$. The frequencies for a single orientation and all orientations are given by

$$
\begin{gathered}
f_{T 6,0}=\frac{f_{T 5,0}}{\tau^{2}}=\frac{1}{5 \tau^{10}}=\frac{89-55 \tau}{5} \simeq 1.626 \times 10^{-3}, \\
f_{T 6}=5 f_{T 6,0}=\frac{1}{\tau^{10}}=89-55 \tau \simeq 8.131 \times 10^{-3} .
\end{gathered}
$$

This total frequency is again larger by a factor of $\tau$ from the value reported in Ref. [29]. This discrepancy is resolved in the next section by showing that the two type-6 LSs sharing the same $S$ vertex are not independent when type-1 to -4 LSs are taken into account.

\section{OVERLAPS AND INDEPENDENCE OF LOCALIZED STATES}

The LSs form a massively degenerate manifold, thus the sum of any two LSs we considered in the previous section is also a LS. We labeled LS eigenstates of the PL vertex model but did not consider if these eigenstates are orthogonal or independent from each other. In this section, we use perpendicular space methods to establish independence of LS types and calculate overlaps between LSs of different types.

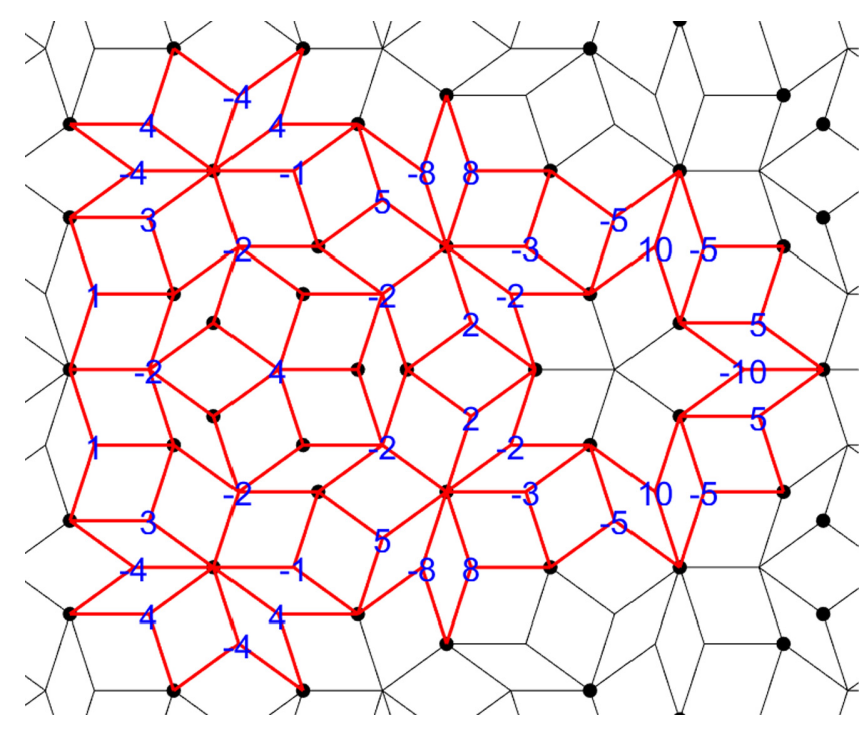

FIG. 17. Type-6 LS has 12 points with index 1 and 29 points with index 3. LS with orientation $\hat{e}_{0}$ is displayed. 

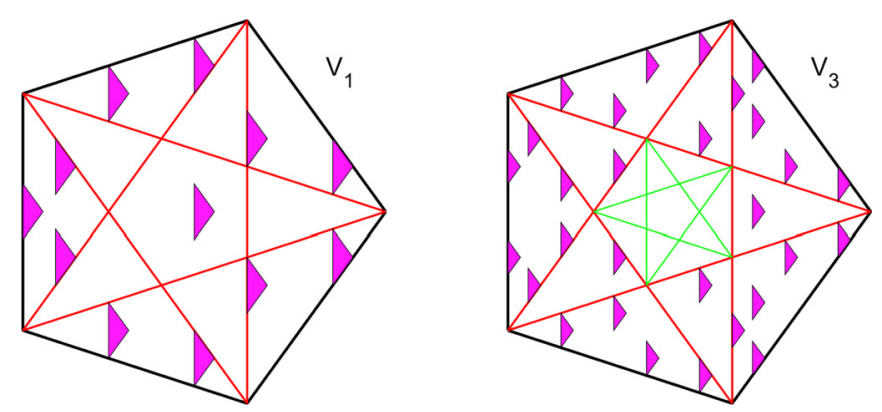

FIG. 18. Allowed areas for type- 6 vertices are triangles which a scaled down by a factor of $\tau$ compared to the allowed areas of type 5 .

The perpendicular space allowed areas for type- 1 states in Fig. 5 show that any two type- 1 states are orthogonal as they correspond to two different points in each pentagonal region. The same logic applied to Fig. 7 yields the orthogonality of any two type- 2 states. However, if we plot the allowed areas for both types simultaneously as in Fig. 20, we observe that there is a significant region of overlap between the two allowed areas. Picking one point in the overlap region and translating it through type- 1 and type- 2 second-neighbor vectors we see that a type- 1 and type-2 LS can share two sites in their support. This situation can be visualized in real space as in Fig. 21. The overlap between the two states can be calculated as

$$
\begin{gathered}
\frac{\left\langle T 1, \vec{R}_{1} \mid T 2, \vec{R}_{2}\right\rangle}{\sqrt{\left\langle T 1, \vec{R}_{1} \mid T 1, \vec{R}_{1}\right\rangle\left\langle T 2, \vec{R}_{2} \mid T 2, \vec{R}_{2}\right\rangle}} \\
=0.2 \sum_{m=0}^{4} \delta\left(\vec{R}_{1}-\vec{R}_{2}-\frac{\hat{e}_{m}}{\tau^{3}}\right) .
\end{gathered}
$$

Investigating the overlap region area in Fig. 20, we see that $\frac{1}{\tau^{4}}=14.59 \%$ of type- 1 states do not have an overlap with type $2, \frac{5}{\tau^{3}\left(1+\tau^{2}\right)}=32.62 \%$ of type- 1 states overlap with only one type- 2 state, while the remaining $\frac{5}{\tau^{2}\left(1+\tau^{2}\right)}=52.79 \%$ have overlaps with two type-2 states. As at most four points of the support in any type- 1 state is covered by type- 2 states all type- 1 and type- 2 states remain linearly independent from each other. Similarly, $\frac{6 \tau-8}{\left(1+\tau^{2}\right)}=47.21 \%$ of type- 2 states do not
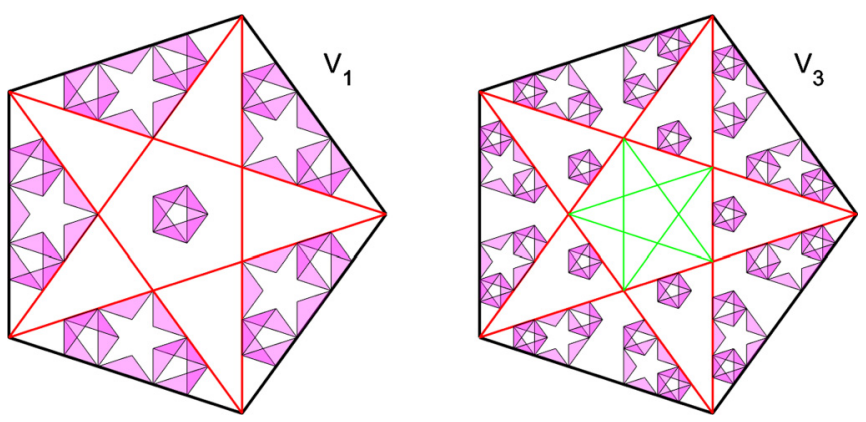

FIG. 19. Type-6 allowed areas for all orientations. Notice that both the central $S$ in $V_{1}$ and the surrounding five $J$ sites in $V_{3}$ can be shared by two type- 6 states with different orientations.

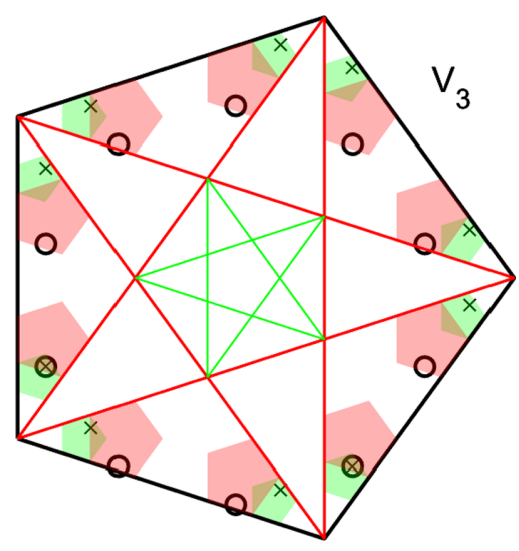

FIG. 20. Perpendicular space overlap of type-1 and type-2 allowed regions. Selecting a point in the overlap region for both type-1 and type- 2 determines the remaining nine points for each LS. Two points out of ten are shared by type- 1 and type- 2 .

have any overlap with type 1 , while $\frac{5}{\tau^{2}\left(1+\tau^{2}\right)}=52.79 \%$ have an overlap with only one type- 1 state.

Type- 3 states are in general not orthogonal to type 1 or type 2. Furthermore, two type-3 states with different orientations can have an overlap as seen in Fig. 10. However, it is easy to establish the linear independence of any type- 3 states from not only types 1 and 2 but also from other orientations of type 3. Investigating the $V_{1}$ pentagon in Figs. 9 and 10, we see the top-left allowed $Q$ region is unique to the $\hat{e}_{0}$ orientation. Similarly, all orientations have unique $Q$ sites which cannot be covered by any sum of type-1, type-2, and other orientations of type- 3 states. Thus, the collection of all type- 1 , type- 2 , and type-3 LSs is a linearly independent but not orthogonal set. In Ref. [28], sum of LSs from this set were defined to be ring

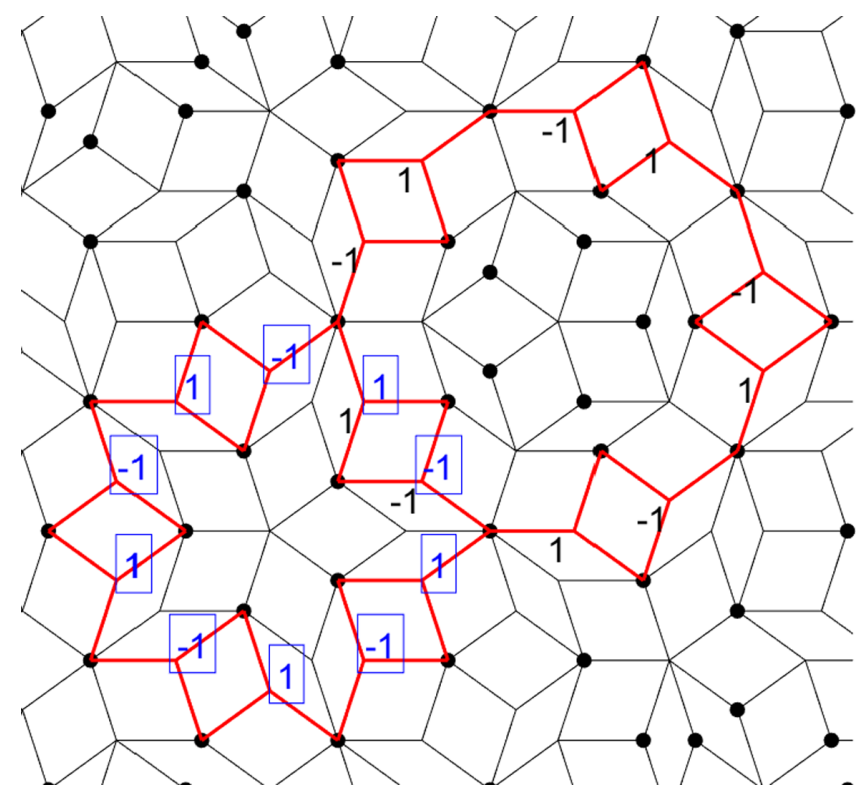

FIG. 21. Real-space images of the points shown in the previous figure. Wave-function values for type 1 are displayed in black (unframed), for type 2 in blue (framed). Overlap of type- 1 and type- 2 states shows that these states are not orthogonal. 

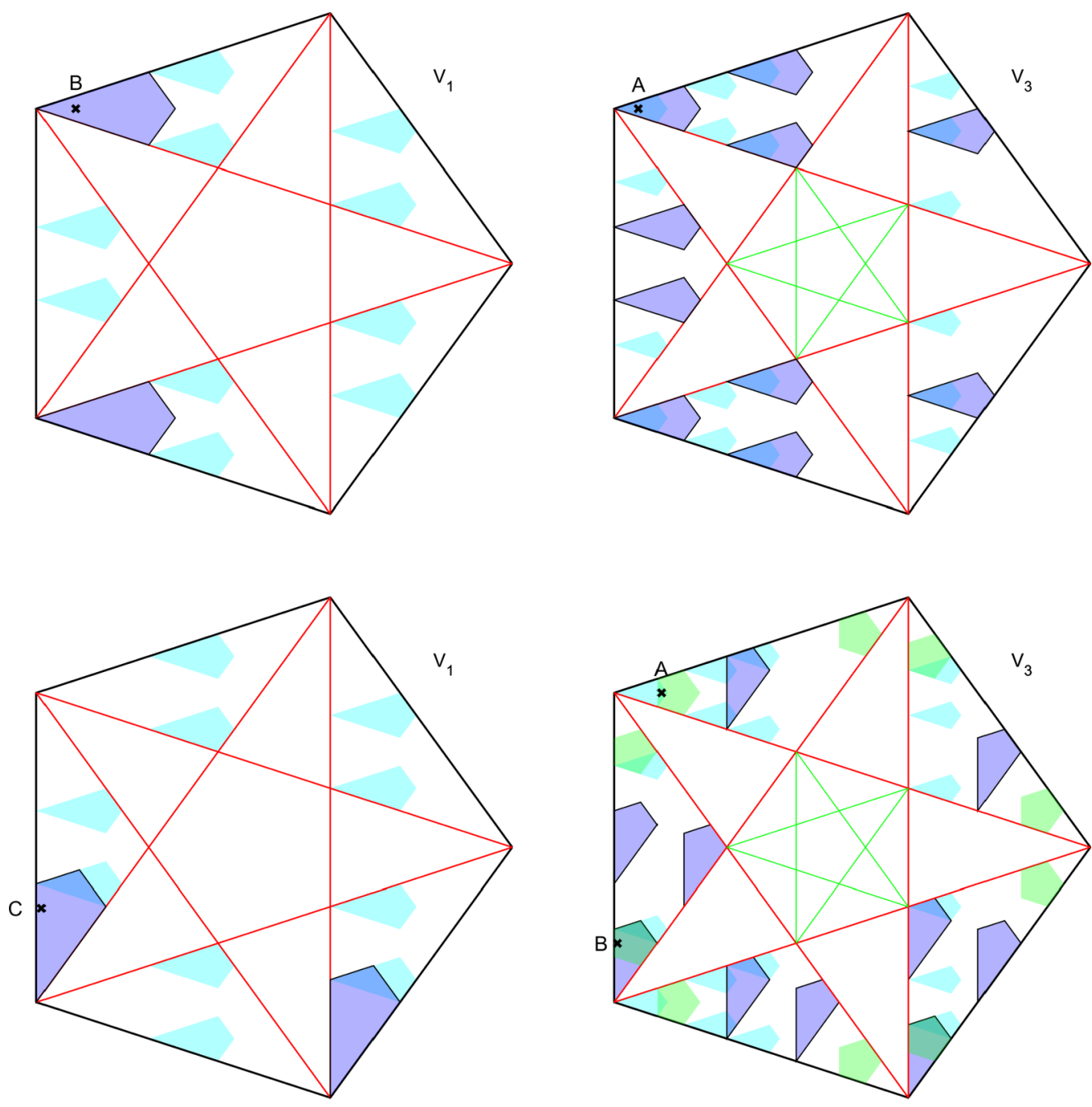

FIG. 22. Perpendicular space images establishing the independence of type-4 LS. Blue, cyan, and green regions correspond to type-4, type-3, and type-1 LSs, respectively. The existence of point A in the upper panel requires point B, which is unique for a single orientation of type-3 LS. In the lower panel, point A is covered by a type-1 state, which creates point B. Point B requires the existence of point C, which is again unique. Thus, type- 4 states of any orientation are linearly independent of all other LS.

states. While it may be possible to construct another basis for the space spanned by types $1-3$, we do not see any advantage to such an approach.

Establishing the independence of type- 4 states requires more work as one cannot find a unique site which is covered only by a type- 4 state of a given orientation. In other words, all the areas in Fig. 12 are covered by either types 1-3 or other orientations of type 4 . To establish independence, we focus on the type- 4 allowed region in the top-left corner of $V_{3}$ as shown in Fig. 22. Point A in this area can only be covered by a type-3 or type- 1 state. If point $A$ is covered by a type- 3 state, this implies the existence of a point $\mathrm{B}$ in $V_{1}$ which is unique to the type- 3 state. As the wave function in point $\mathrm{B}$ cannot be nullified by adding other states and point $\mathrm{B}$ is not in the support of type 4, point A should not be covered by type 3. If point A is covered by a type-1 LS as shown in the lower panels of Fig. 22, this implies the existence of point $\mathrm{B}$ in a different region of $V_{3}$. The amplitude at $\mathrm{B}$ can only be canceled by adding a type-3 state. However, such a type-3 state will generate an amplitude in a unique region of $V_{1}$ at point $C$. Thus, it is impossible to write a type- 4 state as a linear combination of types 1-3 and other type-4 states.

The independence of any type- 5 state from previous types is easily established by noticing that type- 5 states have nonzero amplitude on an $S 5$ site. Thus, the frequency of type-5 states which are independent from all other types is $f_{T 5}=\frac{1}{\tau^{8}}$. This number is a factor of $\tau$ higher than the frequency reported in Ref. [29], a difference which can be discerned with large finite lattice numerical calculations.

It is possible for two type- 5 states of different orientation to have the same $S 5$ point in their support. This can be seen by the overlapping allowed areas in Fig. 16, and picking a point in the allowed region we can visualize the two wave functions in real space: Fig. 23. These two wave functions are obviously independent from each other, and each one of them is independent of all LSs type 1-4. However, the question is if one of the two type-5 states can be written as a sum of the other type-5 state with other LS types. To answer this 


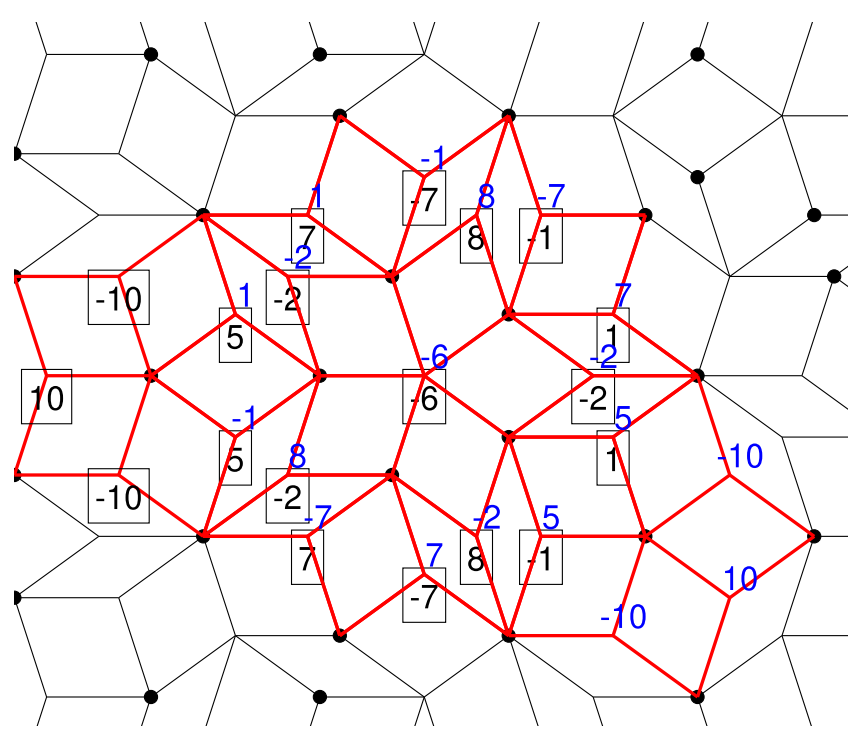

FIG. 23. Type-5 states of two different orientations can share the same $S 5$ vertex and overlap in real space. The wave function values for $\hat{e}_{0}$ orientation are shown in black (framed) and $\hat{e}_{4}$ orientation are shown in blue (unframed).

question, we plot the perpendicular space images of the points in the support of both states in Fig. 24. The two states are not orthogonal:

$$
\begin{aligned}
& \frac{\left\langle T 5, \hat{e}_{0}, \vec{R}_{\perp} \mid T 5, \hat{e}_{4}, \vec{R}_{\perp}\right\rangle}{\sqrt{\left\langle T 5, \hat{e}_{4}, \vec{R}_{\perp} \mid T 5, \hat{e}_{4}, \vec{R}_{\perp}\right\rangle\left\langle T 5, \hat{e}_{0}, \vec{R}_{\perp} \mid T 5, \hat{e}_{0}, \vec{R}_{\perp}\right\rangle}} \\
& =\frac{6}{726} \simeq 0.0008264 .
\end{aligned}
$$

We see that the difference of two wave functions is nonzero on 18 sites. All 18 sites can be covered by a type- 2 state and a type-3 state of a single orientation. Resulting expansion gives us

$$
\begin{aligned}
\left|T 5, \hat{e}_{4}, \vec{R}_{\perp}\right\rangle= & \left|T 5, \hat{e}_{0}, \vec{R}_{\perp}\right\rangle-6\left|T 2, \vec{R}_{\perp}\right\rangle \\
& +10\left|T 3, \hat{e}_{2}, \vec{R}_{\perp}-\frac{\hat{e}_{0}+\hat{e}_{4}}{2 \tau^{3}}\right\rangle
\end{aligned}
$$
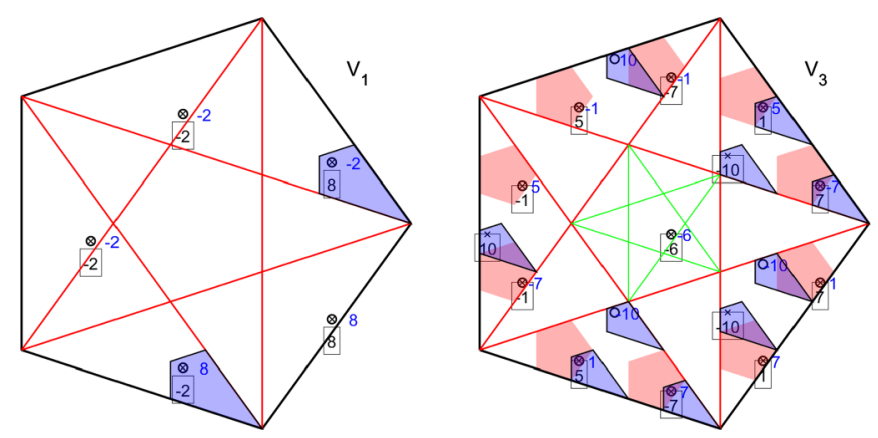

FIG. 24. Perpendicular space image of the two wave functions shown in the previous figure. All the points for which the difference between the wave functions are nonzero can be covered by the allowed areas of type- 3 and type- 2 states as shown. Type- 2 regions are (magenta) pentagons while type- 3 regions are blue kites.

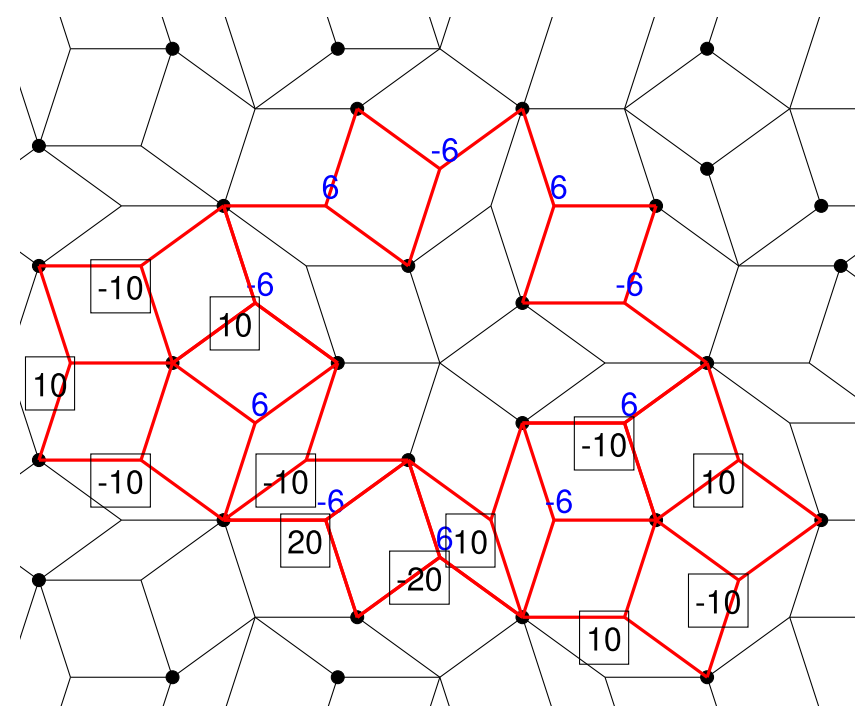

FIG. 25. A type-3 (framed) and a type-2 (unframed) state. The difference of the two wave functions shown in the Fig. 23 is equal to the sum of the wave functions shown in this figure. Different orientations of type-5 are not linearly independent when other types are taken into account.

The expansion can be checked by the real-space wave functions given in Fig. 25.

We see that the two type- 5 states sharing the same $S 5$ site are linearly dependent when other types are taken into account. Any $S 5$ site is in the support of zero, one, or two type-5 LSs, as can be seen by investigating the center of $V_{3}$ in Fig. 16. If we want to count the number of linearly independent type-5 wave functions, we need to consider the same area without any regard to how many times that area is covered. The resulting area is the difference of a pentagon of radius $\tau^{-3}$ and the inside pentagon of radius $\tau^{-5}$ :

$$
f_{T 5}^{\text {indep }}=\frac{\tau^{-6}-\tau^{-10}}{1+\tau^{2}}=\tau^{-9}=\frac{f_{T 5}}{\tau} .
$$

One can form a linearly independent set of LSs by adding one type- 5 orientation per $S 5$ site to the set of all type- 1 to type- 4 states. The frequency of such type- 5 states is given by $f_{T 5}^{\text {indep }}$, which is in agreement with Ref. [29]. The distinction between linearly independent and nonindependent type- 5 states were not made in the literature but it is remarkable that the frequency of the independent type-5 states coincides with the frequency of $S 5$ sites which can host a type-5 state.

A similar scenario plays out for type-6 states. The only LSs which have amplitudes on $S$ and $J$ vertices are type- 6 states, thus each type-6 state is linearly independent of LS types 1-5. However, an $S$ site can host two type-6 states as can be seen in the center of $V_{1}$ in Fig. 19. The real space picture is displayed in Fig. 26 and we see the overlap is

$$
\begin{aligned}
& \frac{\left\langle T 6, \hat{e}_{0}, \vec{R}_{\perp} \mid T 6, \hat{e}_{4}, \vec{R}_{\perp}\right\rangle}{\sqrt{\left\langle T 6, \hat{e}_{4}, \vec{R}_{\perp} \mid T 6, \hat{e}_{4}, \vec{R}_{\perp}\right\rangle\left\langle T 6, \hat{e}_{0}, \vec{R}_{\perp} \mid T 6, \hat{e}_{0}, \vec{R}_{\perp}\right\rangle}} \\
& =-\frac{154}{976} \simeq-0.1578 .
\end{aligned}
$$


While the expansion in terms other LS types is more involved, it can be done systematically by perpendicular space images to yield

$$
\begin{aligned}
\left|T 6, \hat{e}_{0}, \vec{R}_{\perp}\right\rangle-\left|T 6, \hat{e}_{4}, \vec{R}_{\perp}\right\rangle & =12\left|T 1, \vec{R}_{\perp}\right\rangle-5\left|T 2, \vec{R}_{\perp}-\frac{\hat{e}_{0}}{\tau^{3}}\right\rangle-5\left|T 2, \vec{R}_{\perp}-\frac{\hat{e}_{4}}{\tau^{3}}\right\rangle \\
& +10\left|T 4, \hat{e}_{2}, \vec{R}_{\perp}-\frac{\hat{e}_{0}+\hat{e}_{4}}{2 \tau^{4}}\right\rangle-12\left|T 3, \hat{e}_{2}, \vec{R}_{\perp}-\frac{\hat{e}_{2}}{2 \tau^{5}}\right\rangle+8 \sum_{m \neq 2}\left|T 3, \hat{e}_{m}, \vec{R}_{\perp}-\frac{\hat{e}_{m}}{2 \tau^{5}}\right\rangle .
\end{aligned}
$$

The real space contributions of the terms in this expansion are given in Fig. 27. The difference of the two wave functions in Fig. 26 is equal to the sum of all the wave functions in Fig. 27 for each site.

The linear dependence of the two type-6 LSs sharing the same $S$ site reduces the number of independent basis vectors contributed to the LS manifold,

$$
f_{T 6}^{\text {indep }}=\frac{\tau^{-4}-\tau^{-8}}{\tau^{4}\left(1+\tau^{2}\right)}=\tau^{-11}=\frac{f_{T 6}}{\tau},
$$

which is exactly the value reported in Ref. [29], without making a distinction between linearly independent and dependent states.

Considering all the above LS states, we see that certain parts of the perpendicular space are left completely empty. In the next section, we consider sites which are forbidden by local connectivity to host any LS and their perpendicular space images.

\section{FORBIDDEN SITES}

A LS is defined on a set of sites all of which are in the (1-3) sublattice, and all the sites in this set have at least two second-nearest neighbors which are also in this set. Each site in this set, the support of the LS, has a nonzero value of the wave function. Any nearest neighbor of the sites in the support lies in the (2-4) sublattice and defines an equation

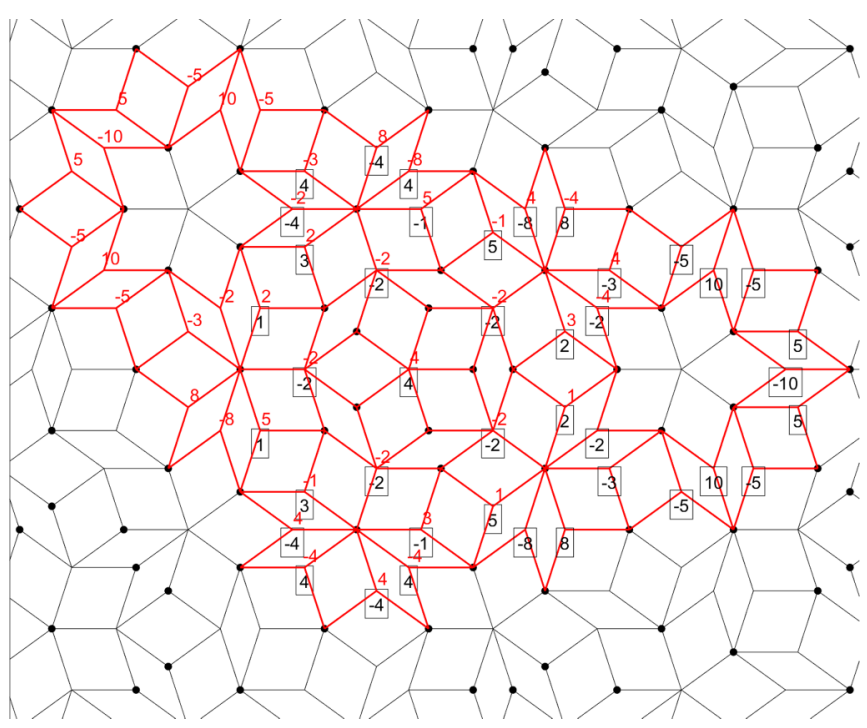

FIG. 26. Type-6 LS of two different orientations overlap in real space. The wave functions of $\hat{e}_{0}$ red (unframed) and $\hat{e}_{4}$ black (framed) are shown. for the values of the wave function on its nearest neighbors. In general, the number of nearest neighbors of any collection of sites is larger than the number of sites in the collection. Thus, generally, the set of equations coming from nearest neighbors define an overdetermined set, and a LS state with such a support becomes impossible. Local connectivity of the PL can cause even further restrictions; there are sites which cannot have a nonzero value of the wave function for any LS. Following Ref. [29], we call such sites forbidden sites and determine the perpendicular space areas associated with them as follows.

First, we consider an $S 5$ vertex with index 2 . All $S 5$ vertices in $V_{2}$ have the same local neighborhood up to their thirdnearest neighbor as shown in Fig. 28. To be more precise, the vertex types of first- and second-nearest neighbors of an $S 5$ in $V_{2}$ are fixed, while only the positions of the third-nearest neighbors are fixed. The sites labeled by A and B in Fig. 28 have more links than the two displayed, and the number and orientation of these links will depend on the position of central $S 5$ in perpendicular space.

As we are considering LSs defined on the (1-3) sublattice, the central $S 5$ and its second-nearest neighbors host no wave function. Instead, the Schrodinger Eq. (8) at each one of these sites give an equation for the wave functions defined in the (1-3) sublattice sites. Let's call the value of the wave function on the five $J$ sites surrounding $S 5 J_{1}, \ldots, J_{5}$ and similarly label the boundary sites with $A_{1}, \ldots, A_{5}$ and $B_{1}, \ldots, B_{5}$. As we are looking for solutions with zero energy, the two equations provided by the sites shown with red diamonds in Fig. 28 are

$$
\begin{array}{r}
A_{5}+J_{5}+B_{3}=0, \\
J_{1}+J_{5}+B_{3}=0 .
\end{array}
$$

These equations are satisfied only if $A_{5}=J_{1}$. Similarly, one can conclude using two other sites that $A_{5}=J_{4}$. Repeating this procedure, we arrive at the conclusion $J_{1}=\ldots=J_{5}=$ $A_{1}=\ldots=A_{5}$. Now the equation provided by the central $S 5$ is $J_{1}+J_{2}+J_{3}+J_{4}+J_{5}=0$, leading to

$$
J_{n}=0, \quad A_{n}=0, \quad n=1, . ., 5,
$$

hence all sites labeled with $J$ and $A$ in Fig. 28 are forbidden sites.

The perpendicular space images for these $J$ and $A$ sites are shown in Fig. 29, where all the pentagons have radius $\tau^{-3}$ originating from the $S 5$ region in $V_{2}$. One can observe that sites labeled with A can be either $K$ or $S$ vertices from $V_{1}$. Now consider the point marked by a red square in Fig. 28. It is a point in the (2-4) sublattice and two of its three neighbors are forbidden by the above argument. Consequently the third neighbor, the site labeled by $\mathrm{B}$, must also be a forbidden site. 


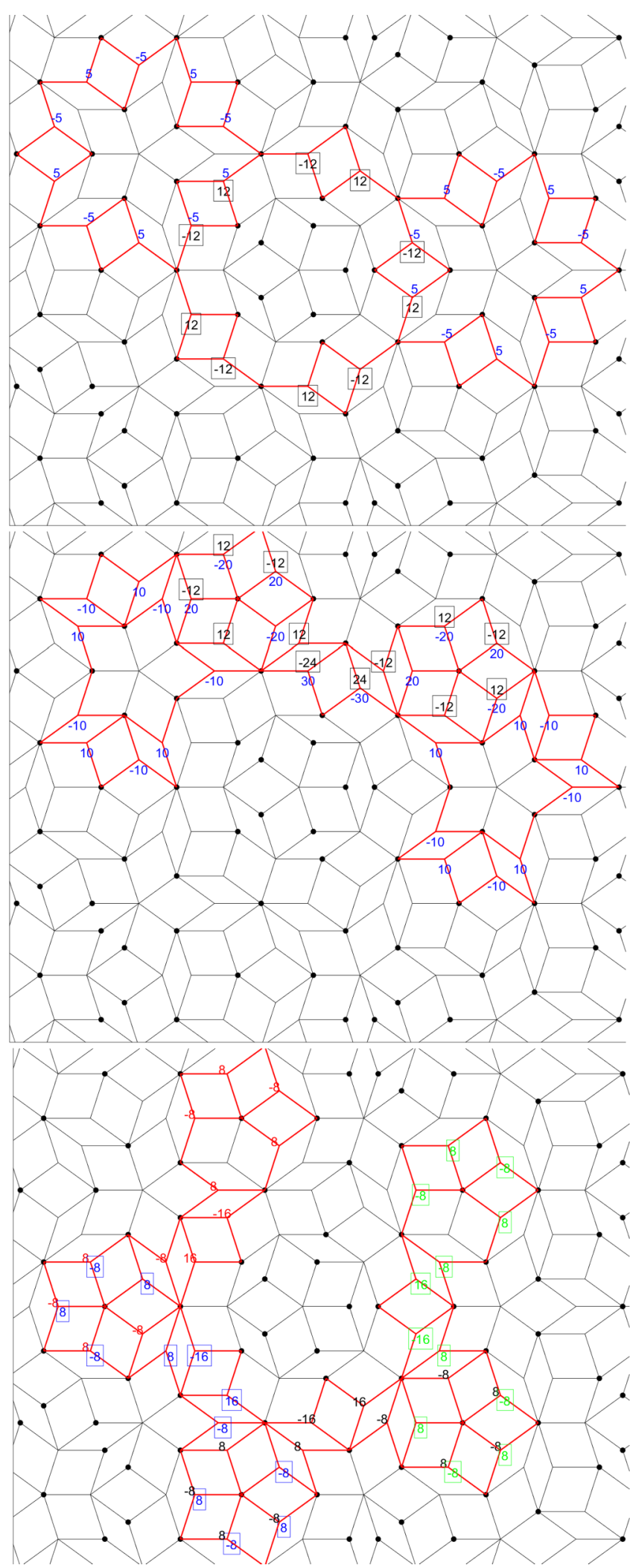

FIG. 27. Real-space wave functions of the terms in Eq. (22). Each panel displays one line on the right-hand side of expansion Eq. (22). The sum of all wave functions shown in the three panels equal the difference of the two wave functions in the previous figure. Two different orientations of type 6 are not linearly independent.

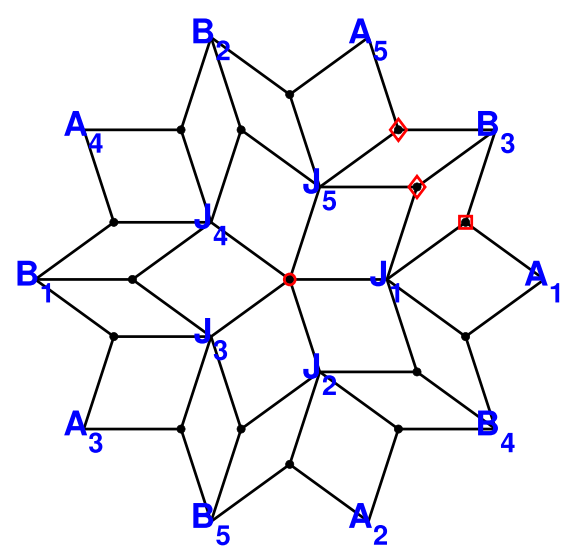

FIG. 28. Local neighborhood up to third-nearest neighbors of an $S 5$ vertex with index 2. All $S 5$ vertices in $V_{2}$ have the same neighborhood; the positions of third-nearest neighbors are fixed but their vertex type can change. Two sites labeled by red diamonds provide equations which require $A_{5}=J_{1}$. The site labeled by the red square requires $B_{3}$ site to be forbidden if $A_{1}$ and $J_{1}$ are forbidden.

More generally, if an $\mathrm{N}$-edge vertex in (2-4) sublattice has $\mathrm{N}-1$ forbidden neighbors, its last remaining neighbor must also be forbidden. This argument can be easily applied in perpendicular space. Consider the forbidden regions shown in Fig. 29; the nearest neighbors of the forbidden sites will define regions in $V_{2}$ and $V_{4}$. Ten pentagons in $\mathrm{D}$ regions of $V_{2}$ and five pentagons in $Q$ regions of $V_{4}$ have two nearest neighbors in the forbidden regions shown in Fig. 30. We can calculate the regions corresponding to the third neighbors of these sites, which come out to be five pentagons shown in Fig. 31. One should note that all $S 4$ vertices are shown to be forbidden sites by this argument, as their regions are completely covered in Fig. 31.

The forbidden regions we have found so far are formed by the union of Figs. 29 and 31. The same algorithm of looking for $N$-edge neighbors with $N$-1 neighbors inside forbidden zones can be iterated. Generally, such seed regions get smaller at each iteration, however, the forbidden region continues to grow until the 15th iteration. At the end of the 15th iteration, the total amount of forbidden regions is plotted in Fig. 32. There are no $N$-edge neighbors with $N$-1 forbidden site neighbors for this region and the iteration process terminates.
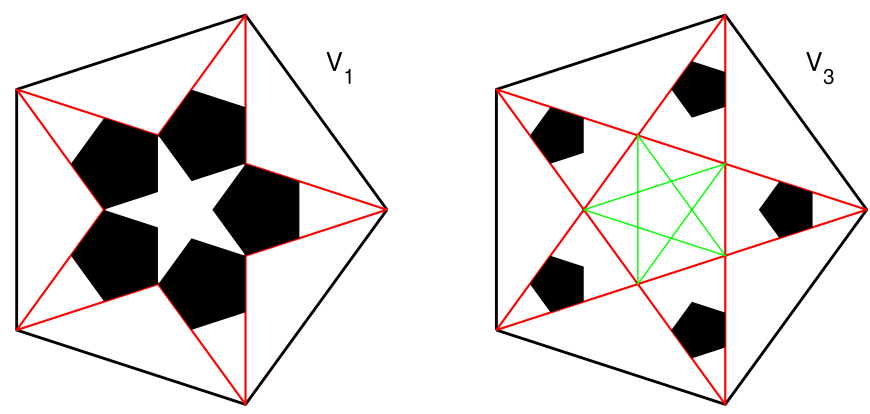

FIG. 29. The forbidden regions in $V_{1}$ and $V_{3}$ corresponding to $J$ and $A$ sites in Fig. 28. We see that $A$ sites can be $S$ or $K$ vertices by comparing with Fig. 2. 

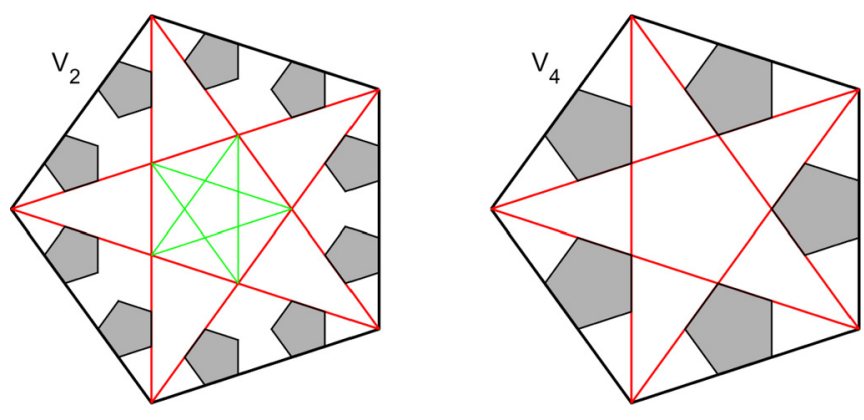

FIG. 30. These seed regions in $V_{2}$ and $V_{4}$ have two nearest neighbors in regions shown in the previous figure. Their remaining nearest neighbors are consequently forbidden.

Now, we consider a similar scenario for the neighborhood of an $S$ vertex with index 4 . The particular neighborhood we consider is plotted in Fig. 33. This neighborhood extending up to the seventh neighbors is unchanged if the central $S$ vertex lies in a pentagon of radius $\tau^{-4}$ at the center of $V_{4}$. Let's take one of the $S 3$ sites in the figure and call the value of the LS wave function on it $A$. By using two $D$ sites sharing the same rhombus, we see that all five $S 3$ sites surrounding the central $S$ must have the same wave function, which are marked with red A's in the figure. The same logic is used to mark the five $K$ sites around the central $S$ and 20 more sites around the five outer $S$ vertices with wave function $A$, shown as blue A's in the figure. The outer $S$ vertices, as well as $J$ sites connecting them have wave function $-2 A$.

The five-nearest neighbors of the central $S$ are marked as $D_{1}, \ldots, D_{5}$. From the central $S$, we have the equation $D_{1}+$ $D_{2}+D_{3}+D_{4}+D_{5}=0$, and the five $J$ sites around the central $S$ give

$$
\begin{aligned}
D_{1}+D_{2} & =D_{2}+D_{3}=D_{3}+D_{4}=D_{4}+D_{5} \\
& =D_{5}+D_{1}=-3 A .
\end{aligned}
$$

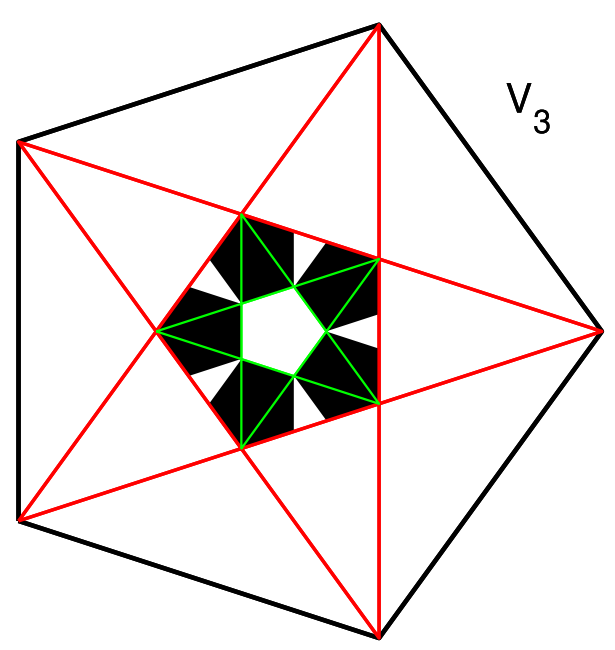

FIG. 31. Perpendicular space forbidden regions which are nearest neighbors of seed regions shown in the previous figure. Notice that all areas corresponding to $S 4$ vertices are covered, thus all S4 vertices are forbidden sites. The process displayed in the last three figures can be iterated.
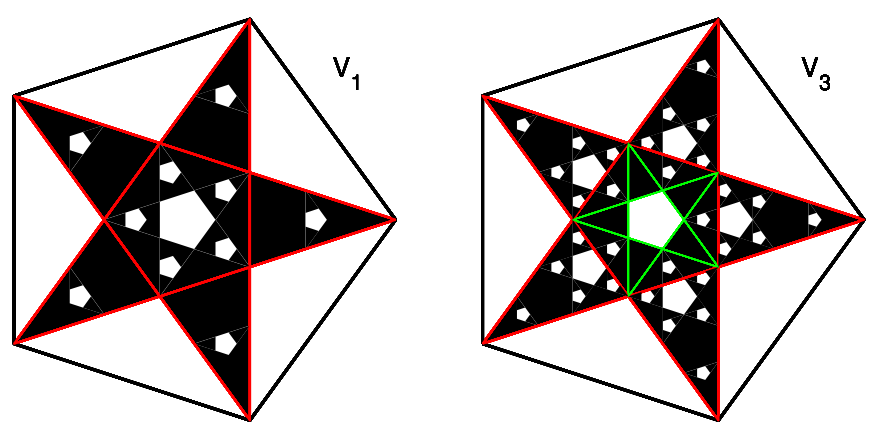

FIG. 32. Forbidden regions found as a result of 15 iterations. The iterations terminate at this stage as there are no $N$ edge vertex regions in $V_{2}$ or $V_{4}$ which have $N-1$ nearest neighbors in the shown forbidden regions.

The only solution to the above set of equations is $A=D_{1}=$ $D_{2}=D_{3}=D_{4}=D_{5}=0$, which shows that all the marked sites in Fig. 33 are forbidden sites. The corresponding forbidden regions in perpendicular space are given in Fig. 34. We can observe that the new forbidden regions fill in some of the holes in Fig. 32. In particular, all $S 3$ vertices are seen to be forbidden sites. If all the allowed regions and the all the forbidden regions above are considered, there are still sites which have not been marked as forbidden or as the support of some LS. We give two more real-space neighborhoods to complete the classification.

We consider the neighborhood of an $S 5$ vertex once again but choose this vertex to lie in $V_{3}$ and hence have a non-zero

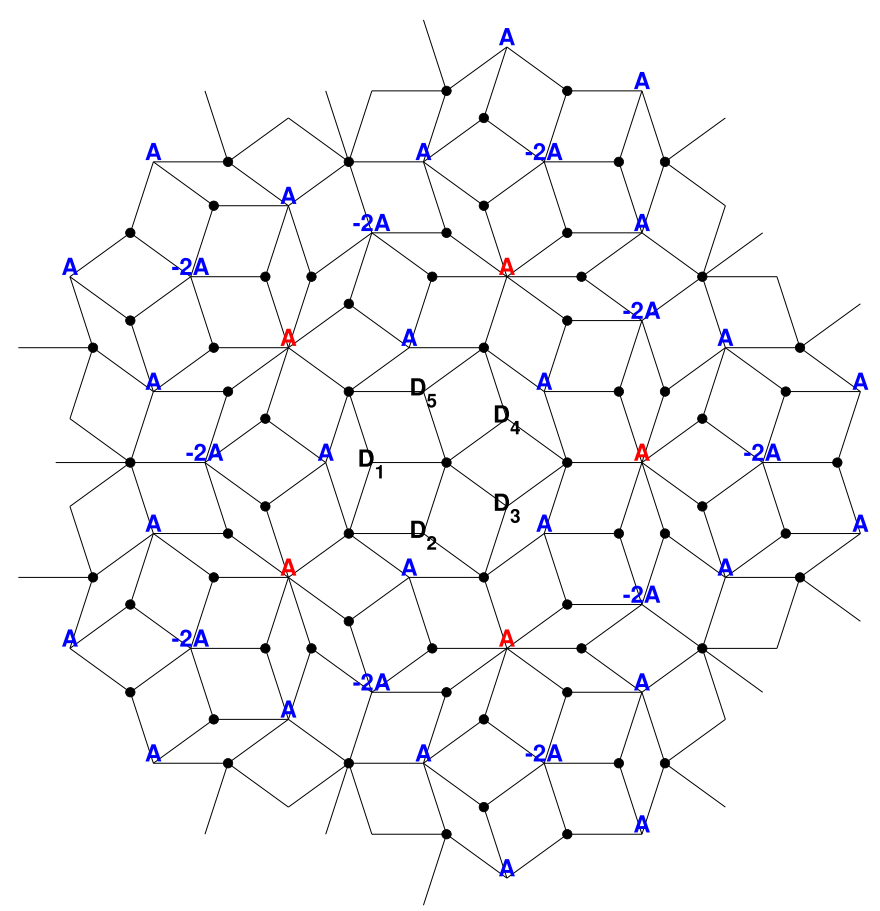

FIG. 33. Neighborhood of an $S$ vertex in $V_{4}$ up to its seventhnearest neighbors. The positions of the sites in this neighborhood are unchanged if $S$ lies in a pentagon of radius $\tau^{-4}$ in perpendicular space. The equations provided by sublattice $(2-4)$ points require all the labeled sites in this figure to be forbidden sites. 

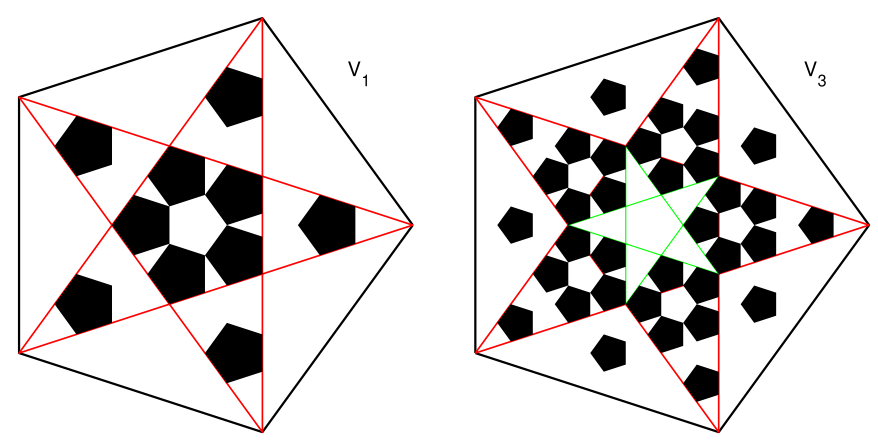

FIG. 34. Forbidden regions corresponding to labeled sites in Fig. 33. Notice that the pentagons in $S 3$ regions close the holes in Fig. 32, making all $S 3$ sites forbidden sites.

wave function. The particular neighborhood we consider is plotted in Fig. 35. Not all $S 5$ sites in $V_{3}$ have the same displayed neighborhood (up to the sixth-nearest neighbors fixed), but $S 5$ must lie in a pentagon of radius $\tau^{-5}$ at the center of $V_{3}$. The sixth neighbors of central $S 5$ contain ten $S 3$ sites, which were proven above to be forbidden. Hence we can mark them with wave function 0 , and mark another smaller ring of zeros using three-edge vertices. The $D$ and $Q$ vertices between this ring and the central $S 5$ are marked with $D_{1}, . ., D_{10}$ and $Q_{1}, \ldots, Q 5$. The first set of equations come from $K$ sites,

$$
\begin{aligned}
D_{2}+D_{3} & =D_{4}+D_{5}=D_{6}+D_{7}=D_{8}+D_{9} \\
& =D_{10}+D_{1}=0,
\end{aligned}
$$

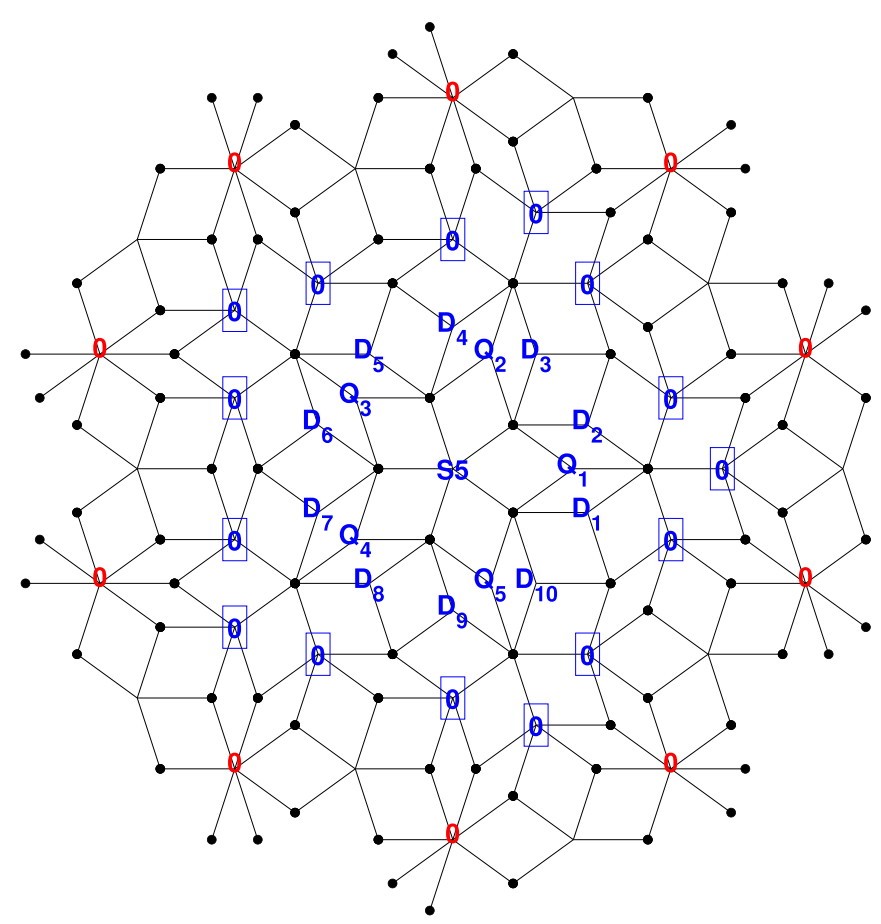

FIG. 35. Local neighborhood of an $S 5$ site in $V_{3}$ up to seventhnearest neighbors. The positions of the sites in the figure are fixed if the $S 5$ vertex lies in a pentagon of radius $\tau^{-5}$ at the center of perpendicular space.
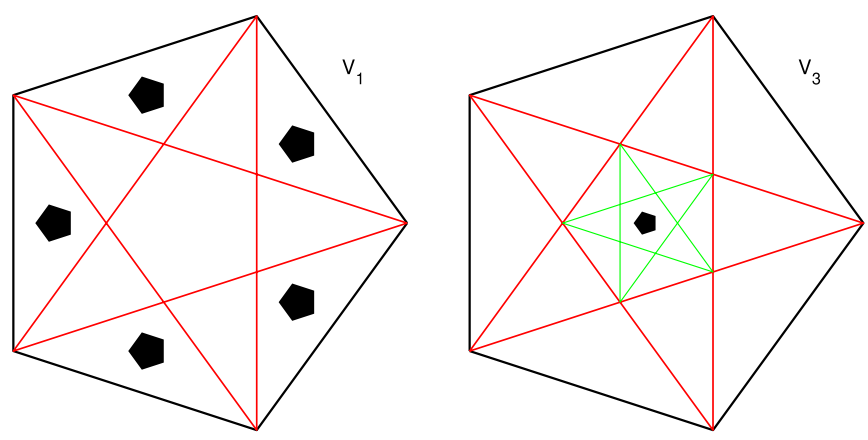

FIG. 36. Forbidden regions corresponding to $Q$ and $S 5$ labels in Fig. 35.

followed by

$$
\begin{aligned}
D_{2}+D_{3}+Q_{1}+Q_{2} & =-S 5, \\
D_{4}+D_{5}+Q_{2}+Q_{3} & =-S 5, \\
D_{6}+D_{7}+Q_{3}+Q_{4} & =-S 5, \\
D_{8}+D_{9}+Q_{4}+Q_{5} & =-S 5, \\
D_{10}+D_{1}+Q_{5}+Q_{1} & =-S 5 .
\end{aligned}
$$

This tells us all $Q$ sites must have the same wave function, which is $-1 / 2$ of the central $S 5$. Finally, using the $S 4$ site equations,

$$
\begin{gathered}
D_{1}+D_{2}+Q_{1}=0, \\
D_{3}+D_{4}+Q_{2}=0, \\
D_{5}+D_{6}+Q_{3}=0, \\
D_{7}+D_{8}+Q_{4}=0, \\
D_{9}+D_{10}+Q_{5}=0,
\end{gathered}
$$

we conclude both the central S5 and the five Q surrounding it are forbidden sites. The perpendicular space regions corresponding to these sites are given in Fig. 36.

The final real-space configuration we consider is given in Fig. 37, consisting of the ten nearest neighbors of an $S$ site in $V_{1}$. This neighborhood is fixed if the central $S$ lies in a pentagon of radius $\tau^{-6}$ at the center of $V_{1}$.

The five $J$ sites surrounding the center must have the same wave function, which is $-1 / 2$ of the wave function of the central $S$. Eighth neighbors of $S$ have $S 3$ vertices which we can mark with zero wave function as they were proven to be forbidden above. This allows us to mark a number of sixth and tenth neighbors with zero wave function, forming an outside ring. This ring leads us to denote $15 D$ sites with $\pm D_{1}, . . \pm D_{5}$. All the remaining sites we mark with $x_{1}, . . x_{30}$.

The equations coming from $S 3$ sites give us

$$
x_{1}+x_{2}+\ldots+x_{30}=-5 J,
$$

but $J$ site equations similar to $x_{2}+x_{3}+x_{4}+x_{5}=0$ allow us to reduce this sum to $\left(x_{30}+x_{1}\right)+\left(x_{6}+x_{7}\right)+$ $\left(x_{12}+x_{13}\right)+\left(x_{18}+x_{19}\right)+\left(x_{24}+x_{25}\right)=-5 J$. We then find $D_{1}+D_{2}+D_{3}+D_{4}+D_{5}=-5 J$. The sum in Eq. (30) can be reduced in another way using $K$ site equations like $x_{3}+x_{4}=-2 J$ and $J$ site equations like $x_{1}+x_{2}=D_{1}$, 


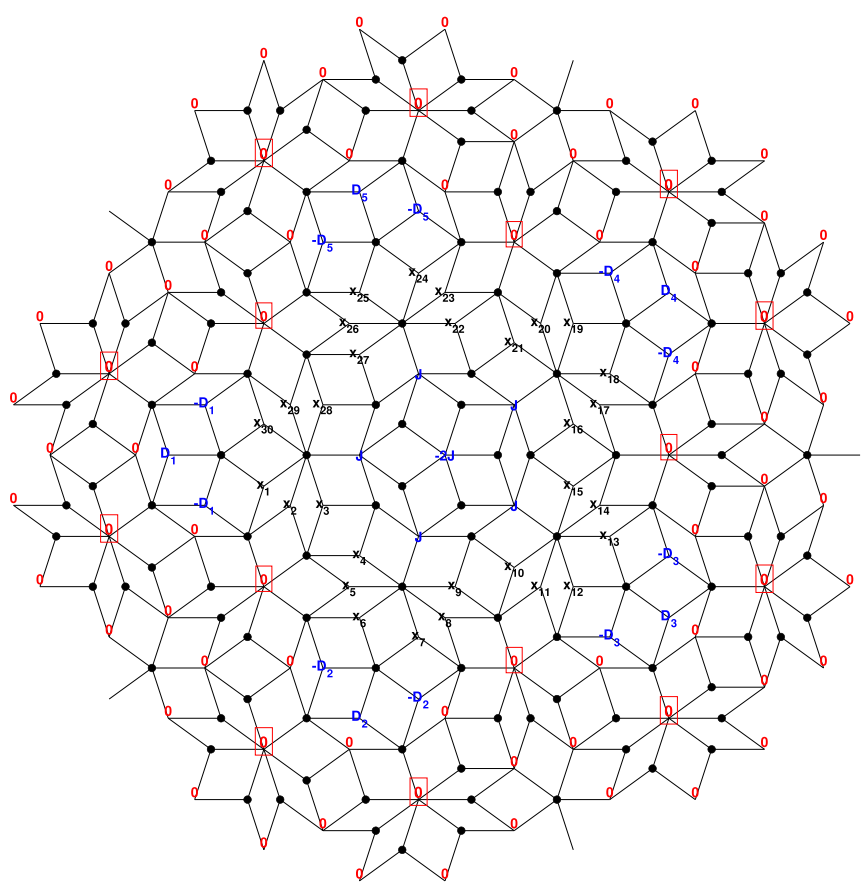

FIG. 37. Local neighborhood up to tenth-nearest neighbors of an $S$ vertex of index 1 . The central $S$ site lies in a pentagon of radius $\tau^{-6}$ at the center of perpendicular space. Central $S$ and five surrounding $J$ sites are forbidden as explained in the text.

yielding

$$
\begin{aligned}
\left(x_{1}+x_{2}\right)+\left(x_{3}+x_{4}\right)+\ldots+\left(x_{29}+x_{30}\right) & =-5 J,(31) \\
D_{1}-2 J+\ldots+D_{1}=2\left(D_{1}+\ldots+D 5\right)-10 J & =-5 J .
\end{aligned}
$$

However, as we found $D_{1}+D_{2}+D_{3}+D_{4}+D_{5}=-5 J$, the equation becomes $20 J=5 J$, where the only solution is $J=0$. Thus we mark the central $S$ and surrounding $J$ regions in $V_{1}$ and $V_{3}$ as forbidden in Fig. 38.

When we overlay all the allowed regions in Figs. 5, 7, $10,13,16,19$, and all the forbidden regions in Figs. 32, 34, 35, and 38, we obtain Fig. 39. There are no areas which are not assigned either as forbidden or as in the support of some LS. This constitutes geometric proof that a site is either forbidden to host a LS or is in the support of some LS. Overall $f_{\text {forbidden }}=1358-839 \tau \simeq 46.95 \%$ of PL vertices cannot host a LS.
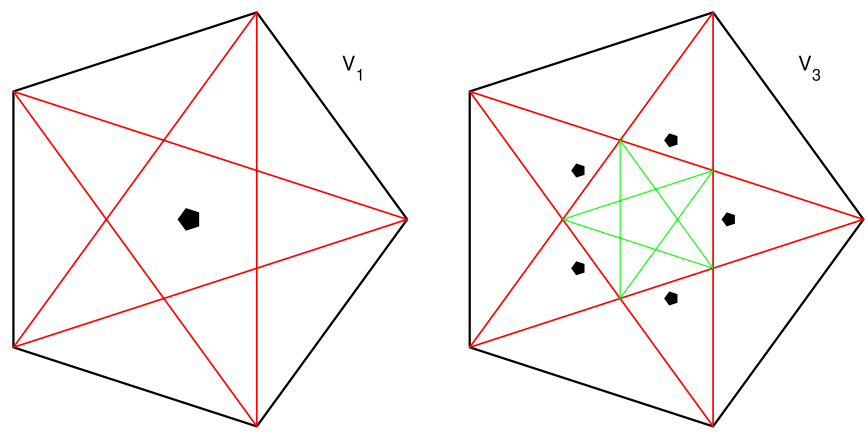

FIG. 38. Perpendicular space regions for forbidden sites in Fig. 37.
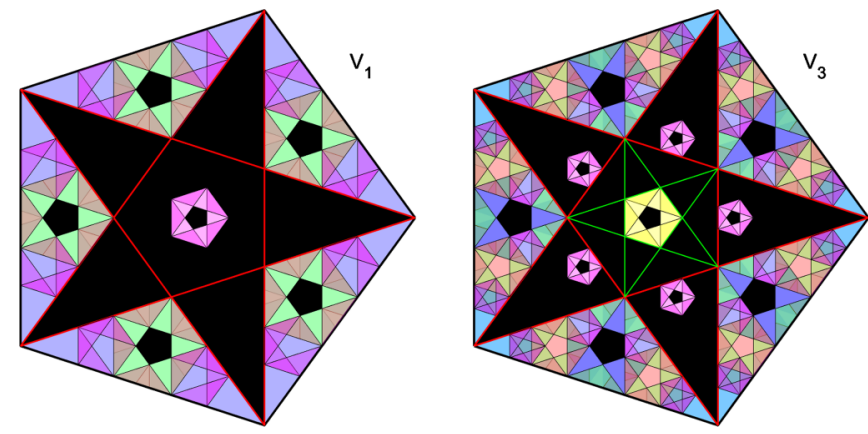

FIG. 39. All the forbidden regions (Figs. 32, 35, 34, and 38) and allowed regions (Figs. 5, 7, 10, 13, 16, and 19) displayed. All areas inside the four perpendicular space pentagons are filled, thus any site in the PL is either forbidden or in the support of at least one LS.

\section{CONCLUSION}

Quasicrystal lattices can be defined as projections of higher dimensional periodic structures. All points in the real-space lattice have an image in the projected-out dimensions, and this perpendicular space image carries valuable information about the local neighborhood in real space. All the images in perpendicular space lie inside a finite region, and this region is filled densely and uniformly. These properties make it possible to use perpendicular space to label and count local structures in real space.

We applied these perpendicular space accounting methods to label and count the LS in the vertex tight-binding model of the PL. We verified the frequencies of type- 1 to type- 4 LSs but showed that the number of type- 5 and type- 6 LS that can be defined on the PL are a factor of $\tau$ larger than previously reported values. We proved the independence of different types of states and showed how perpendicular space methods can be used to calculate overlaps between them. This allowed us to show that the frequency of linearly independent type-5 and type-6 LS is equal to previously reported values for the total number of these states. We also proved that any site in the PL is either in the support of at least one LS or forbidden by local connectivity to host a LS. It has been conjectured that the six types of LS states span the whole zero-energy manifold of the vertex tight-binding model. We have not been able to prove this but our analysis shows that if another LS state exists it has to be defined on sites which are in the support of type-1 to type-6 LSs. Thus, such a LS cannot be trivially independent by having a unique site in its support.

The perpendicular space method is independent of the scaling (inflation-deflation) symmetry of the PL, which is generally used to count local structures. It would be interesting to see if this method can be combined with scaling symmetries as done for the Fibonacci chain [20] to investigate eigenstates which are not strictly localized. Similarly, other models defined on quasicrystals [17,21] which have strictly localized excitations may benefit from a perpendicular space approach.

\section{ACKNOWLEDGMENT}

M.M. is supported by the Bilkent Comprehensive Undergraduate Scholarship. 
[1] D. Shechtman, I. Blech, D. Gratias, and J. W. Cahn, Metallic Phase with Long-Range Orientational Order and no Translational Symmetry, Phys. Rev. Lett. 53, 1951 (1984).

[2] V. Elser and C. L. Henley, Crystal and Quasicrystal Structures in Al-Mn-Si Alloys, Phys. Rev. Lett. 55, 2883 (1985).

[3] P. A. Kalugin, A. Yu. Kitayev, and L. S. Levitov, 6-dimensional properties of Al0.86Mn0.14 alloy, J. Phys. Lett. 46, 601 (1985).

[4] D. Levine and P. J. Steinhardt, Quasicrystals. I. Definition and structure, Phys. Rev. B 34, 596 (1986).

[5] J. E. S. Socolar and P. J. Steinhardt, Quasicrystals. II. Unit-cell configurations, Phys. Rev. B 34, 617 (1986).

[6] W. Steurer, Quasicrystals: What do We Know? What do We Want to Know? What can We Know? Acta Crystallogr. Sec. A 74, 1 (2018).

[7] M. Kohmoto, L. P. Kadanoff, and C. Tang, Localization Problem in One Dimension: Mapping and Escape, Phys. Rev. Lett. 50, 1870 (1983).

[8] S. Ostlund, R. Pandit, D. Rand, H. J. Schellnhuber, and E. D. Siggia, One-Dimensional Schrödinger Equation with an Almost Periodic Potential, Phys. Rev. Lett. 50, 1873 (1983).

[9] M. Kohmoto, B. Sutherland, and C. Tang, Critical wave functions and a cantor-set spectrum of a one-dimensional quasicrystal model, Phys. Rev. B 35, 1020 (1987).

[10] H. Tsunetsugu, T. Fujiwara, K. Ueda, and T. Tokihiro, Electronic properties of the Penrose lattice. I. Energy spectrum and wave functions, Phys. Rev. B 43, 8879 (1991).

[11] Z. V. Vardeny, A. Nahata, and A. Agrawal, Optics of photonic quasicrystals, Nat. Photonics 7, 177 (2013).

[12] D. Tanese, E. Gurevich, F. Baboux, T. Jacqmin, A. Lemaître, E. Galopin, I. Sagnes, A. Amo, J. Bloch, and E. Akkermans, Fractal Energy Spectrum of a Polariton Gas in a Fibonacci Quasiperiodic Potential, Phys. Rev. Lett. 112, 146404 (2014).

[13] K. Singh, K. Saha, S. A. Parameswaran, and D. M. Weld, Fibonacci optical lattices for tunable quantum quasicrystals, Phys. Rev. A 92, 063426 (2015).

[14] K. Viebahn, M. Sbroscia, E. Carter, J.-C. Yu, and U. Schneider, Matter-Wave Diffraction from a Quasicrystalline Optical Lattice, Phys. Rev. Lett. 122, 110404 (2019).

[15] K. Kamiya, T. Takeuchi, N. Kabeya, N. Wada, T. Ishimasa, A. Ochiai, K. Deguchi, K. Imura, and N. K. Sato, Discovery of superconductivity in quasicrystal, Nat. Commun. 9, 154 (2018).

[16] S. Sakai, N. Takemori, A. Koga, and R. Arita, Superconductivity on a quasiperiodic lattice: Extended-to-localized crossover of cooper pairs, Phys. Rev. B 95, 024509 (2017).
[17] F. Flicker, S. H. Simon, and S. A. Parameswaran, Classical Dimers on Penrose Tilings, Phys. Rev. X 10, 011005 (2020).

[18] N. G. de Bruijn, Algebraic theory of Penrose's non-periodic tilings of the plane. I, Indagationes Math. (Proc.) 84, 39 (1981).

[19] S. Hoffmann and H.-R. Trebin, Rows in two-dimensional quasilattices, Phys. Status Solidi B 174, 309 (1992).

[20] N. Macé, A. Jagannathan, and F. Piéchon, Fractal dimensions of wave functions and local spectral measures on the Fibonacci chain, Phys. Rev. B 93, 205153 (2016).

[21] A. Szallas and A. Jagannathan, Spin waves and local magnetizations on the Penrose tiling, Phys. Rev. B 77, 104427 (2008).

[22] P. Kalugin and A. Katz, Electrons in deterministic quasicrystalline potentials and hidden conserved quantities, J. Phys. A: Math. Theore. 47, 315206 (2014).

[23] N. Macé, A. Jagannathan, P. Kalugin, R. Mosseri, and F. Piéchon, Critical eigenstates and their properties in one- and two-dimensional quasicrystals, Phys. Rev. B 96, 045138 (2017).

[24] Katz, A. and Duneau, M., Quasiperiodic patterns and icosahedral symmetry, J. Phys. France 47, 181 (1986).

[25] R. Penrose, The role of aesthetics in pure and applied mathematical research, Bull. Inst. Math. Appl. 10, 266 (1974).

[26] T. Odagaki and D. Nguyen, Electronic and vibrational spectra of two-dimensional quasicrystals, Phys. Rev. B 33, 2184 (1986).

[27] T. C. Choy, Density of States for a Two-Dimensional Penrose Lattice: Evidence of a Strong Van-Hove Singularity, Phys. Rev. Lett. 55, 2915 (1985).

[28] M. Kohmoto and B. Sutherland, Electronic States on a Penrose Lattice, Phys. Rev. Lett. 56, 2740 (1986).

[29] M. Arai, T. Tokihiro, T. Fujiwara, and M. Kohmoto, Strictly localized states on a two-dimensional Penrose lattice, Phys. Rev. B 38, 1621 (1988).

[30] V. Kumar, D. Sahoo, and G. Athithan, Characterization and decoration of the two-dimensional Penrose lattice, Phys. Rev. B 34, 6924 (1986).

[31] C. L. Henley, Sphere packings and local environments in Penrose tilings, Phys. Rev. B 34, 797 (1986).

[32] M. V. Jarić, Diffraction from quasicrystals: Geometric structure factor, Phys. Rev. B 34, 4685 (1986).

[33] M. Baake, Quasicrystals: An Introduction to Structure, Physical Properties and Applications, 1st ed. (Springer, Berlin, 2002) Chap. 2, p. 17.

[34] J. Vidal, R. Mosseri, and B. Douçot, Aharonov-Bohm Cages in Two-Dimensional Structures, Phys. Rev. Lett. 81, 5888 (1998). 